\title{
FIVE SHORT STORIES ABOUT THE CARDINAL SERIES
}

\author{
BY J. R. HIGGINS
}

\section{CONTENTS}

Introduction

Story One. Historical notes

Story Two. Some methods for deriving the cardinal and allied series

Story Three. $L^{2}$ and $L^{p}$ theory

Story Four. The cardinal series and LCA groups

Story Five. Extensions to higher dimensions

Conclusion

\section{INTRODUCTION}

Suppose that a function $g$ generates a Fourier series in the usual way:

$$
g(x) \sim \sum c_{n} e^{-i n x}
$$

Now multiply both members by $e^{i x t} / 2 \pi$ and formally integrate over a period. On the right we obtain

$$
\sum c_{n} \frac{\sin \pi(t-n)}{\pi(t-n)}
$$

or, equivalently,

$$
\frac{\sin \pi t}{\pi} \sum c_{n} \frac{(-1)^{n}}{t-n}
$$

which is called a "cardinal series". On the left we obtain a function $f$ whose form

$$
f(t)=\frac{1}{2 \pi} \int_{-\pi}^{\pi} g(x) e^{i x t} d x
$$

suggests that it has a Fourier transform with compact support on $[-\pi, \pi]$, or, put another way, $f$ has no frequency content outside the "band" $[-\pi, \pi]$. One can expect that such an $f$ will be represented in some sense by the cardinal series (1), and that in all likelihood the coefficient $c_{n}$ will, because of (2), be of the form $f(n)$.

Received by the editors September 19, 1984.

1980 Mathematics Subject Classification. Primary 41A05, 42C10; Secondary 41-03, 01A55, 42B99, 42C30, 94-03, 01A60, 94A05. 
The purpose of this article is to bring under review some of the mathematics surrounding the cardinal series, including a few notes on its history. Some of the material seems to be new, but the presentation will be largely expository in character.

A major factor affecting current interest in the cardinal series is its importance for certain applications; in fact, its use as an essential ingredient in the sampling theory of band-limited signals in communications engineering constitutes a truly important piece of scientific methodology. During the past three decades or so, this application alone has provided a strong impetus to further the mathematical theory, much of which has in fact appeared in engineering literature.

This sampling principle has even begun to penetrate the semipopular literature; see, e.g., Bigelow and Day (1983, p. 102), where it appears in a rather unexpected context.

There are two recent survey articles in this area. A. J. Jerri's (1977) covers a wide range of topics and has a large and useful bibliography; it is firmly oriented towards applications and includes results on stochastic processes. $P$. L. Butzer's (1983) is more modest in its scope and aims to survey some of the approximation theory associated with the cardinal series, with particular emphasis on those parts developed by himself and his colleagues at Aachen during the past decade.

I certainly have neither the intention nor the competence to vie with either of these surveys; but I think that the more purely mathematical theory of cardinal series and its historical origins deserve a fuller account than has been attempted to date. I shall try to do this by drawing on the whole of the historical range of material and on engineering, as well as mathematical, literature.

I. Kluvánek (1965) has remarked that "The origin of this theorem [the sampling theorem below] can hardly be traced". In the first of the stories to follow, which charts a historical journey starting in nineteenth-century France, I hope to show that this is an unduly pessimistic assertion. The first explicit mention of this sampling theorem (part A) and the cardinal series known to me occurs in three works of E. Borel dating from the last years of that century (see $\S 1.1$ for a fuller account). These contributions of Borel, as well as those of several other people, seem to have been overlooked in contemporary sources. Certain mysteries begin to appear $(\$ \S 1.2,1.3)$ as we penetrate back further into the nineteenth century in search of deeper roots.

I should add that the "history" will be of the rather superficial "who knew what and when" kind. Much of what we subsequently cover will be seen to have its origins in this historical material.

These later stories can be left to speak for themselves. I make no apology for including so many examples in $\$ 4.2$, most of which have not been pointed out before; in total, they show what a powerful unifying principle Kluvánek's theorem (\$4.1) is in this area, and there seems to be something interesting to say about each one. There are also some other items of novelty, mostly consisting of "infilling", which include the complete orthonormal character of the set in Item 2, §3.1, and the main theorem on absolute convergence in $\$ 3.4$. 
Before the storytelling begins I want to describe in slightly more detail how the cardinal series is applied in the sampling theory of band-limited signals (those functions having the property described in part B below), and for this purpose I can do no better than turn to the seminal paper of C. E. Shannon (1949).

In this paper Shannon gives a presentation of the sampling theorem with which his name has become closely associated (although we shall see in $\$ 1.5$ that he was preceded in this). This sampling theorem should really be considered in two separate parts, and we set these out below as parts A and B. Later, we allow a looser usage of the phrase "sampling theorem" and use it to mean an assertion about the representation of a function $f$, band-limited in some sense, by a series generated from some set of data associated with $f$.

SAMPling TheOREM (PART A). If a function $f(t)$ contains no frequencies higher than $W / 2$ cycles per second, it is completely determined by giving its ordinates at a sequence of points spaced $1 / W$ seconds apart.

Sampling Theorem (Part B). A function $f(t)$ band-limited to $[-\pi W, \pi W]$, i.e., with the form

$$
f(t)=\int_{-\pi W}^{\pi W} g(x) e^{i x t} d x,
$$

is the sum of its "scaled" cardinal series

$$
f(t)=\sum_{n=-\infty}^{\infty} f\left(\frac{n}{W}\right) \frac{\sin \pi(W t-n)}{\pi(W t-n)} .
$$

Shannon was able to say of part A that it was "common knowledge in the communications art", and this knowledge was based on the following intuitive justification: if $f$ contains no frequency higher than $W / 2 \mathrm{cps}$, it cannot change to a substantially new value in a time less than half a cycle of this highest frequency, that is, $1 / W$ seconds.

Shannon then proceeded to put the matter on a somewhat firmer footing, arguing something like this: since the general Fourier coefficient of $g$ (in (3)) is $f(n / W)$, the ensemble of "samples" $\{f(n / W)\}, n=0, \pm 1, \ldots$, determines $g$ via its Fourier series, and $g$ in turn determines $f$ via (3).

As to part B, Shannon's justification runs more or less like this: the sum of the cardinal series on the right in (4) is band-limited to $[-\pi W, \pi W]$ since this is true of each term separately (one shows this using elementary properties of the Fourier transform). Also, if $m$ and $n$ are integers, the "interpolatory" property

$$
\frac{\sin \pi(W t-n)}{\pi(W t-n)}= \begin{cases}0, & t=m / W, m \neq n, \\ 1, & t=n / W,\end{cases}
$$

shows that this sum coincides with $f$ at the sample points. Hence, by part $\mathrm{A}$ the sum is none other than $f$ itself.

Of course there are other ways of reconstructing $f$ from its sample values; for example, one can use the Newton-Gauss interpolation series (see, e.g., J. M. 
Whittaker (1935, p. 62)). However, it is the cardinlal series which has found favour in signal-processing applications, undoubtedly because of the neat way in which it fits into the accompanying Fourier analysis.

It was in this way that Shannon established the following important engineering principle: if a signal has bounded frequency content, then all the information contained in that signal is in fact contained in the sample values at equidistantly spaced sample points, and knowledge of the bound determines the minimum rate at which the signal needs to be sampled in order to reconstruct it exactly. This rate, $W$ samples per second, is called the "Nyquist sampling rate" (see Shannon $(1949$, p. 12) for references to early work of Nyquist and others in this field).

Interestingly enough, Shannon goes on to mention that other sets of data can also be used to determine the band-limited signal $f$ : for example, the values of $f$ and its first derivative at every other sample point, the values of $f$ and its first and second derivatives at every third sample point, and so on. Also, it is possible to use sample points that are not equidistantly spaced. Several years were to elapse before mathematical formulations of these procedures were developed (see, e.g., §§2.3, 3.1, 3.2).

In a further interesting section, Shannon gives what amounts to a Hilbert space formulation of the problem of transmitting a message over a band-limited communication channel and even includes a little glossary, of eleven items, comparing engineering and vector space terminologies.

It should be mentioned that these remarks were all made in relation to signals of finite duration; it was J. D. Weston who gave the complete Hilbert space formulation of the situation, independently of Shannon and at about the same time (see $\$ 1.5)$.

Notations are mostly standard. The Fourier transform of $f$,

$$
\mathscr{F} f(x)=\frac{1}{\sqrt{2 \pi}} \int_{\mathbb{R}} f(t) e^{-i x t} d t
$$

where $\mathbb{R}$ denotes the real numbers, will often be denoted $\hat{f}$, and $f^{\sim}$ will denote the inverse transform. A naked summation sign means that the index $n$ is to range over the integers $\mathbb{Z}$ or over $\mathbb{Z}^{k}$ as the context demands; a prime ' denotes the omission from summation of the term corresponding to $n=0$; convergence is understood in the sense of spherical partial sums.

\section{STORY ONE}

\section{HISTORICAL NOTES}

1.1. Beginnings. The cardinal series can be obtained formally by considering the Lagrange interpolation formula in the form

$$
H_{m}(z)\left\{\frac{f(0)}{z}+\sum_{n=1}^{m}\left[\frac{f(n)}{H_{m}^{\prime}(n)(z-n)}+\frac{f(-n)}{H_{m}^{\prime}(-n)(z+n)}\right]\right\},
$$


where

$$
H_{m}(z)=z \prod_{n=1}^{m}\left(1-\frac{z^{2}}{n^{2}}\right),
$$

which interpolates a function $f(z)$ at the points $z=-m, \ldots, 0, \ldots, m$. Since

$$
\frac{\sin \pi z}{\pi z}=\prod_{n=1}^{\infty}\left(1-\frac{z^{2}}{n^{2}}\right)
$$

the cardinal series is obtained by letting $m \rightarrow \infty$ (see T. A. Brown (1915-1916) and Ferrar (1925, p. 270); see also J. M. Whittaker (1935, p. 63) where a more general limiting procedure is carried out). It can also be obtained formally as a special case of Cauchy's partial fractions expansion for a suitably restricted meromorphic function $F$ with poles at the points $\left(p_{n}\right)$, namely

$$
F(z)=\sum \text { residue at } w=p_{n} \text { of } \frac{F(w)}{(z-w)} ;
$$

one applies this to $F(z)=f(z) / \sin \pi z$, where $f$ is entire, and the cardinal series (1b) results (see Ferrar (1925, p. 281)).

The first explicit use of the cardinal series which I have been able to discover is in a brief note by Borel (1898, p. 1002), who was discussing the question of how the power series coefficients $\left(a_{n}\right)$ of a function $f(z)=\sum a_{n} z^{n}$ determine its singularities. One way of getting information on this is to form an auxiliary function $\psi$ determined (in part) by the conditions $\psi(n)=a_{n}$; from the many ways of doing this, Borel chose

$$
\psi(z)=\frac{\sin \pi z}{\pi} \sum_{n=0}^{\infty} \frac{a_{n}}{z-n},
$$

with $\sum\left|a_{n}\right|<\infty$ for convergence. This certainly has the appearance of the cardinal series (1b), but on closer inspection we notice that the summation does not extend over negative values of $n$, and the factor $(-1)^{n}$ is missing from the summand. No matter, the next year Borel (1899) returned to the interpolation problem, set $a_{n}=a_{-n}$ and used the full cardinal series expansion, complete with $(-1)^{n}$ in the summand, under the less restrictive convergence criterion

$$
\sum\left|\frac{a_{n}}{n}\right|<\infty
$$

This is an important inequality in the theory of cardinal series, and we shall return to it in \$3.4. Borel went on to give a more general form of the series with the factor $(z / n)^{p}$ in the summand, a device for improving the convergence behaviour (p. 85). He also mentioned (p. 83) that he deduced the series from Lagrange's interpolation formula.

A couple of years earlier, Borel (1897) had been studying the general Lagrange-type formula

$$
f(z)=\sum \frac{c_{n} \phi(z)}{\phi^{\prime}\left(a_{n}\right)\left(z-a_{n}\right)},
$$


with

$$
\sum\left|\frac{c_{n}}{a_{n} \phi^{\prime}\left(a_{n}\right)}\right|<\infty,
$$

for convergence, which he had also studied in his thesis. The significant passage here is on p. 675:

\section{Posons}

$$
f(z)=\int_{-\pi}^{\pi} \psi(x) e^{i z x} d x
$$

et supposons que la fonction $\psi(x)$ satisfasse aux conditions de Dirichlet. Dès lors, si l'on connait les valeurs de $f(z)$ pour $z=$ $0, \pm 1, \pm 2, \ldots$, la fonction $\psi(x)$ est déterminée et par suite la fonction entière $f(z)$ est connue sans ambiguïté.

This leaves no doubt that what we here call Sampling Theorem A for band-limited functions was known to Borel at this time. It is interesting to note that his justification for it is virtually the same as Shannon's (see the Introduction). I must point out, however, that nowhere can I discover that Borel linked his statement of Sampling Theorem A with his use of the cardinal series, which would have established Sampling Theorem B.

A few years later Hadamard (1901) made a much more extensive study of this same problem of determining properties of a function from its power series coefficients. He used the same formula as Borel for the auxiliary function (quoting only Borel's 1898 paper and still omitting $(-1)^{n}$ ), but he too gave the less restrictive convergence condition (6).

An interpolation scheme due to de la Vallée Poussin (1908) is often quoted as being an early form of the sampling theorem; actually it would be more realistic to describe it as a close relative. De la Vallée Poussin considered (op. cit., p. 227) the finite interpolation formula

$$
\frac{\sin m t}{m} \sum_{a}^{b}(-1)^{n} \frac{f(n \pi / m)}{t-n \pi / m},
$$

where $f$ is a given function defined on $[a, b]$, and the summation is understood to be over those $n$ for which $n \pi / m \in[a, b)$. The limit $m \rightarrow \infty$ is now taken, and de la Vallée Poussin's main result (p. 341) is that the formula converges to $f(t)$ at any point $t$ in a neighbourhood of which $f$ is continuous and of bounded variation.

This kind of interpolation has come back into fashion during the last decade or so, largely as a result of the need to apply sampling theorems to durationlimited, as opposed to band-limited, signals (see, e.g., Butzer $(1983, \S 3)$ ).

Ferrar (1926) reported that de la Vallée Poussin's work had been applied and extended by Steffensen (1914) and Theis (1919). This is true of Theis, as her title suggests, but Steffensen's main source for the cardinal series was Hadamard (op. cit.), and he referred to de la Vallée Poussin only in passing. Steffensen (p. 83) seems to have been the first to relate the cardinal series to other interpolation series, in this case Newton's divided difference formula. 
1.2. The Cauchy connection. In this section I want to mention the first of two mysteries concerning the early history of the cardinal series which I am presently unable to resolve.

This concerns a passage from H. S. Black (1953, p. 41) which is worth quoting in full:

If a signal is a magnitude-time function, and if time is divided into equal parts forming subintervals such that each subdivision comprises an interval $T$ seconds long where $T$ is less than half the period of the highest significant frequency component of the signal; and if one instantaneous sample is taken from each subinterval in any manner; then a knowledge of the instantaneous magnitude of each sample plus a knowledge of the instant within each subinterval at which the sample is taken contains all of the information of the original signal.

This is followed by a reference to Cauchy (1841). I must report that I have been unable to find anything to support such a statement in this reference or, indeed, in any other of Cauchy's writings. Following Black, later writers have given the same reference, however, and suggested that "...the sampling theorem was already known to Cauchy..." (Yen (1956); see also Petersen (1963) and Haddad et al. (1967); Jerri (1977, p. 1565) sidesteps the issue by saying "...some attribute it [the sampling theorem] to Cauchy..." and refers to Black).

Cauchy's paper is in two parts. By a strange coincidence the first part is concerned with developing certain finite interpolation formulae (by taking the interpolation points in Lagrange's formula to be in geometric progression), which have every right to be called cardinal series; but we shall have to look at the abstract harmonic analysis setting (\$4.2) before being able to see this, and it seems impossible that these were what Black was referring to. The second part of Cauchy's paper is concerned with trigonometric interpolation and is quoted in historical studies (e.g., Burkhardt (1899-1916, p. 642 et seq.)) as being one of the main sources for this. The difficulty is that Cauchy's paper is about finite interpolation and contains no reference to band-limited functions, as required in the quotation above.

The intention behind this reference remains a mystery.

1.3. The Poisson connection. Unresolved mystery number two concerns the following description of the sampling theorem by Neveu $(1965$, p. 50): “... le théorème d'échantillonnage dit de Shannon mais dû en fait à Poisson". No reference is given, and I have not been able to find any definite evidence for either part of the sampling theorem in the works of Poisson. Certainly the cardinal series is closely connected to Poisson's summation formula, but this seems to be a much later development (see \$2.5).

At the same time, in searching through Poisson's collected works I did come across a most interesting paper (1820) on the vibrations of a string composed of two different parts. 
Let us take up the story with Poisson on p. 460 , where we find a pair of coupled nonharmonic Fourier sine expansions:

$$
\begin{aligned}
& f(x)=\sum B_{\lambda} \sin \lambda \frac{L^{\prime}}{a^{\prime}} \sin \lambda \frac{x}{a}, \\
& g\left(x^{\prime}\right)=\sum B_{\lambda} \sin \lambda \frac{L}{a} \sin \lambda \frac{x^{\prime}}{a^{\prime}} ;
\end{aligned}
$$

here the summations are taken over the positive roots $\left(\lambda_{n}\right)$ of

$$
P(\lambda) \equiv a^{\prime} \sin \frac{\lambda L^{\prime}}{a^{\prime}} \cos \frac{\lambda L}{a}+a \cos \frac{\lambda L^{\prime}}{a^{\prime}} \sin \frac{\lambda L}{a}=0,
$$

$f$ and $g$ represent the initial states of the two pieces of string, $L$ and $L^{\prime}$ are their lengths, and $x^{\prime}=L+L^{\prime}-x$. The coupling is due to Poisson's initial conditions.

With Poisson we multiply the first of these coupled equations by $\sin (t x / a)$ and integrate over $(0, L)$; we multiply the second by $\sin \left(t x^{\prime} / a^{\prime}\right)$ and integrate over $\left(0, L^{\prime}\right)$, and then add the results; after some reduction one obtains

$$
F(t)=P(t) \sum_{ \pm \lambda_{n}} \frac{F\left(\lambda_{n}\right)}{P^{\prime}\left(\lambda_{n}\right)\left(\lambda_{n}-t\right)},
$$

in which

$$
\begin{aligned}
F(t)= & \frac{a^{\prime}}{a} \sin \frac{t L^{\prime}}{a^{\prime}} \int_{0}^{L} \sin \frac{t x}{a} f(x) d x \\
& +\frac{a}{a^{\prime}} \sin \frac{t L}{a} \int_{0}^{L^{\prime}} \sin \frac{t x^{\prime}}{a^{\prime}} g\left(x^{\prime}\right) d x^{\prime} .
\end{aligned}
$$

Because of the special nature of the sum $F$, this is more than just another Lagrange interpolation series. The case $a^{\prime}=a=L^{\prime}=L=1$ reduces to what is effectively the cardinal series representation for a function $F$ which is even and such that $F(t) / \sin t$ is band-limited.

This raises the question of whether we can say that Poisson discovered the sampling theorem for band-limited functions in 1820. On the strength of these calculations one might be inclined to say yes, but at this point I must confess to having prejudiced the issue to some extent. Where I have written the variable $t$, Poisson had $\lambda^{\prime}$, which he used along with $\lambda$ to represent any root of $P(\lambda)=0$, and his intention was merely to find the coefficients $\left(B_{\lambda}\right)$ in (7) (which he does on p. 462). Thus, with hindsight the interpolation series (8) is read into Poisson's presentation without difficulty; of course, I have done this because it seems a rather interesting situation in its own right, but, all in all, one can hardly credit Poisson with something he did not intend.

So a second mystery remains.

1.4. From E. T. Whittaker to G. H. Hardy. In this section we shall concentrate on some of the interesting material from the interwars period which seems to have fallen into an undeserved oblivion. This historical period is, for our purposes, conveniently delimited by two landmark studies in the theory of cardinal series. 
The first of these is the groundbreaking study by E. T. Whittaker (1915). It contains no references of any kind and really represents a new beginning. Whittaker posed the familiar interpolation problem of finding a function which passes through the points $\left(a+n w, f_{n}\right), a, w$ complex, $n=0, \pm 1, \pm 2, \ldots$. He called the class of all such functions the cotabular set associated with $\left(f_{n}\right)$ and showed that the sum of what we now call the cardinal series picks out a special member, the "cardinal" function, of this cotabular set. Whittaker's new idea was that this function is special because it is entire and free from "violent oscillations", or, more precisely, it has "no constituents whose period is less than twice the tabular interval w" (p. 193); in other words, it is band-limited. Then Whittaker's series (op. cit., p. 186) for the cardinal function $f$ takes the form

$$
\sum f(a+n w) \frac{\sin \pi(x-a-n w) / w}{\pi(x-a-n w) / w} .
$$

He did not call it the cardinal series-the name seems to first appear in the works of J. M. Whittaker, his second son, about 1920.

Before exploring further in this historical period, it should be mentioned that, according to Ferrar (1926, p. 333), F. J. W. Whipple, in an unpublished manuscript dated 1910, introduced the cardinal series somewhat earlier than E. T. Whittaker and discovered several of its properties, including the bandlimited nature of its sum, which he called slowly swinging. It is tempting to think that Whipple was familiar with these lines from "Seal Lullaby" in Rudyard Kipling's The jungle book:

The storm shall not wake thee nor shark overtake thee, Asleep in the arms of the slow-swinging seas.

Incidentally, it was Whipple who coined the phrase "well poised" for a certain kind of hypergeometric series, a field in which he is much better known. It is unclear why he preferred not to publish his results on the cardinal series.

We come now to one of the highlights of this period-the use of cardinal series, and certain extensions, to deduce properties of entire functions from their known behaviour at a sequence of points. There are one- and two-dimensional results of this kind.

In order to approach these ideas let $f$ be entire, of exponential type $\pi$, and bounded over $\mathbb{R}$. Then $(f(z)-f(0)) / z$ is also of exponential type $\pi$ and belongs to $L^{2}(\mathbb{R})$. Now a function satisfying these conditions is represented pointwise over $\mathbb{R}$ by its cardinal series (see $\mathrm{H} 1$ et seq.). After writing out the appropriate series and rearranging it (using the partial fractions expansion for $\csc \pi z$ ), one gets

$$
f(z)=\frac{\sin \pi z}{\pi}\left\{f^{\prime}(0)+\frac{f(0)}{z}+\Sigma^{\prime}(-1)^{n} f(n)\left[\frac{1}{n}+\frac{1}{z-n}\right]\right\} .
$$

Note that, as well as the sampled values of $f$ at the integers, one item of derivative information is also required for the reconstruction of $f$. Here, the 
more usual $L^{2}$ condition is replaced with one of boundedness over $\mathbb{R}$; consequently, $f$ need not be band-limited (but see the further remarks about this series in Topic II of the Conclusion). The series (9) was introduced by Tschakaloff (1933), who used it to give an affirmative answer to a problem posed by Pólya (1931):

Let $f(z) e^{-\varepsilon z}$ be bounded over the complex plane for each $\varepsilon>0$.

Does the boundedness of the sequence $(f(n)), n=0, \pm 1, \ldots$, imply that $f$ is constant?

We might call (9) the $T$-cardinal series. It was used shortly after this by Cartwright (1936) and Macintyre (1938) to prove theorems on growth properties of entire functions. Macintyre gave a second extension along the same lines:

$$
\begin{aligned}
f(z)= & \frac{z \sin \pi z}{2 \pi} f^{\prime \prime}(0)+\frac{\sin \pi z}{\pi} f^{\prime}(0) \\
& +\frac{\sin \pi z}{\pi}\left\{\left[\frac{1}{z}+\frac{\pi^{2} z}{3 !}\right] f(0)+\sum^{\prime} \frac{(-1)^{n} z^{2} f(n)}{n^{2}(z-n)}\right\} .
\end{aligned}
$$

Here, $f$ is still of exponential type $\pi$ but need only be $O(|x|)$ over $\mathbb{R}$. We can call this the $T_{2}$-cardinal series.

Macintyre also mentioned that the ordinary, the $T$ - and the $T_{2}$-cardinal series are all special cases of a much more general class of interpolation series discussed some years earlier by G. Valiron (1925). Let $\mu$ be a nonnegative integer, $F$ an entire function (called the base function), and $p_{k}$ a polynomial of degree $k$. Then Valiron calls

$$
F(z)=\sum \frac{k_{n} z^{\mu}}{a_{n}^{\mu} F^{\prime}\left(a_{n}\right)\left(z-a_{n}\right)}+p_{\mu-1}(z)
$$

the "Lagrange interpolation series of rank $\mu$ ", convergent if

$$
\sum \frac{k_{n}}{F^{\prime}\left(a_{n}\right) a_{n}^{\mu+1}}<\infty .
$$

Clearly we get the ordinary, $T$ - and $T_{2}$-cardinal series by taking $\sin \pi z$ for the base function, $\mu=0,1,2$, respectively, etc. Also it will be obvious how to construct a $T_{k}$-cardinal series for a function of exponential type $\pi$ which is $O\left(|x|^{k}\right)$ over $\mathbb{R}$ (see, e.g., Boas $(1954$, p. 221$)$ ).

On looking into Valiron's paper one finds that he gives no references for (10), and his general tone seems to indicate that he expected his readership to be familiar with it. In fact, he was not the originator, and it does go back much further in the literature; see P. Cazzaniga (1882) and M. Guichard (1884) for early versions; also see P. E. B. Jourdain (1905). Nevertheless, Valiron's name seems to have become firmly linked with the series (e.g., Macintyre (1938) and R. P. Boas (1954, p. 232)).

Zygmund (1959, p. 276) gives a somewhat different application of the $T$-cardinal series, using it to prove Bernstein's theorem on entire functions of exponential type and deducing some interesting summation formulae. 
The $T$-cardinal series was discovered independently by Hardy (see $\mathrm{H} 6$ of this section) and M. Zakai (see Conclusion, Topic II). More recently, it has been studied from the point of view of approximation theory by W. Splettstösser et al. (1981), who call it the interpolating series of Valiron; it has also been used by D. H. Mugler (1976), who attributes it to Macintyre.

At about the same time that Tschakaloff was solving Pólya's problem, Pólya himself was contributing to the solution of a rather similar two-dimensional version of it. J. E. Littlewood had conjectured that:

If $f$ is an entire function of order less than two, then the boundedness of the double sequence $(f(m+i n))(m, n=$ $0, \pm 1, \ldots)$ implies that $f$ is constant.

It was J. M. Whittaker (see (1935, p. 73)) who first settled this conjecture in the affirmative, and to do it he introduced the following two-dimensional form of the cardinal series.

THEOREM. Let $f$ be entire such that

$$
\varlimsup_{r \rightarrow \infty} \frac{\log M(r)}{r^{2}}<\frac{\pi}{2}
$$

where $M$ is, as usual, the maximum modulus function. Then

$$
f(z)=\sigma(z) \sum f(m+i n) \frac{(-1)^{m+n+m n} \exp \left\{-\pi\left(m^{2}+n^{2}\right) / 2\right\}}{z-m-i n} .
$$

Note that the part played by $\sin \pi z$ in the ordinary cardinal series (1b) is now taken over by the Weierstrass sigma function $\sigma(z)$, which has zeros at the "lattice points" $m+$ in.

However, it was Pólya who noticed that Littlewood's conjecture falls out from (11) in a couple of lines or so (see J. M. Whittaker (1935, p. 73)).

Shortly afterwards, this two-dimensional cardinal series appeared in one or two studies concerned with similar problems (see J. M. Whittaker (1935, p. 104) for references), but after that it seems to have been completely forgotten. It would be interesting to know something more about it: for example, whether or not the expansion functions have any nice orthogonality or completeness properties.

Spain (1940) ingeniously used the cardinal series to interpolate a sequence, not of function values, but of operators $\left(D^{n}\right)$; here $D^{n}$ denotes $n$-fold differentiation if $n$ is a positive integer, $n$-fold integration if $n$ is a negative integer, and the identity operator if $n$ is zero. This process was considered somewhat disappointing in that it did not produce the usual fractional derivative operator (but this operator is produced (Spain (1958)) if the Newton-Gregory interpolation formula is used instead).

We end our survey of the present historical period with an important study by G. H. Hardy (1941). It is self-indulgent perhaps, but I cannot resist quoting six items from Hardy's treasure chest of beautiful results on cardinal series.

First, we recall that the classical Paley-Wiener theorem asserts that the class of functions which are entire, of exponential type $T$, and whose restrictions to 
the real axis belong to $L^{2}(\mathbb{R})$ is identical to the class of functions $f$ with a representation

$$
f(z)=\int_{-T}^{T} g(u) e^{i z u} d u, \quad g \in L^{2}(-T, T) .
$$

Thus, Hardy (1941, p. 332) found it natural to call members of this class Paley-Wiener functions. Note that these Paley-Wiener functions form a Hilbert subspace of $L^{2}(\mathbb{R})$; we shall denote it $\mathrm{PW}_{T}$, or just $\mathrm{PW}$ if $T=\pi$.

H1. The functions

$$
w(t, n) \equiv \frac{\sin \pi(t-n)}{\pi(t-n)}, \quad n=0, \pm 1, \ldots,
$$

form a complete orthonormal set in $\mathrm{PW}$.

Shannon (1949, p. 13) mentioned the orthogonality without proof; Hardy, on the other hand, gave two proofs. One uses contour integration. The other, which gives the completeness as well, proceeds from the complete orthonormal character of $\left\{e^{i n x} / 2 \pi\right\}$ in $L^{2}(-\pi, \pi)$ via the Fourier transform (see also §3.1). $\mathrm{H} 1$ has been rediscovered many times since Hardy's day.

Hardy also mentioned that the expansion for $f \in \mathrm{PW}$ in the set $\{w(t, n)\}$ is its cardinal series and converges uniformly on compact subsets of $\mathbb{R}$.

H2. PW is a reproducing kernel Hilbert space. The reproducing kernel is

$$
w(t, x) \equiv \frac{\sin \pi(t-x)}{\pi(t-x)} .
$$

(Hardy did not use the phrase "reproducing kernel".) Thus, the inner product of $f \in \mathrm{PW}$ with $w$ reproduces $f$ :

$$
f(t)=\int_{\mathbb{R}} f(x) \frac{\sin \pi(t-x)}{\pi(t-x)} d x .
$$

This integral equation is ascribed by Hardy (p. 340) to H. Bateman (see also, Titchmarsh (1948, p. 349)).

It is of interest to note that every $f \in \mathrm{PW}$ can be represented as a convolution sum (its cardinal series) and as a convolution integral (12). The similarity between these two representations is striking (and can be generalised; see §3.2). Indeed, the integral can be thought of as a "continuous analogue" of the series; Boas and Pollard (1973) have given several interesting examples of this kind of analogue. $f$ set

Hardy went on to discuss function classes more general than $L^{2}(\mathbb{R})$. For any

$$
F(t)=\int_{\mathbf{R}} f(x) w(t, x) d x .
$$

$F$ is frequently encountered in Hardy's theorems. If $f$ does belong to $L^{2}(\mathbb{R})$ then $F$ is the orthogonal projection of $f$ on PW. It is natural to consider the Fourier series for $f$ in the set $\{w(t, n)\}$ :

$$
\sum c_{n} w(t, n)
$$


where

$$
c_{n}=\int_{\mathbf{R}} f(t) w(t, n) d t .
$$

If this series converges in some sense, it does not necessarily do so to $f$, of course; it can be the cardinal series for $F$ (see, e.g., H4 below).

DEFINITIONS. Let

$$
\begin{gathered}
\mathscr{M}=\left\{f: \int_{\mathbf{R}} \frac{|f(x)|}{2+|x|} d x<\infty\right\}, \\
\mathscr{M}^{*}=\left\{f: \int_{\mathbf{R}}|f(x)| \frac{\log (2+|x|)}{2+|x|} d x<\infty\right\} .
\end{gathered}
$$

We have $L^{2}(\mathbb{R}) \subset \mathscr{M}^{*} \subset \mathscr{M}$.

H3. If $f \in \mathscr{M}^{*}$ (resp. $\mathscr{M}$ ) then (13) converges (resp. is $C 1$ summable) to $F$ uniformly on compact subsets of $\mathbb{R}$.

DeFinitions. Let

$$
\mathscr{P}=\{f: f=F\}, \quad \mathscr{B}=\mathscr{M} \cap \mathscr{P} .
$$

H4. Let $f \in \mathscr{M}$; then the Fourier series in $\{w(t, n)\}$ for $f$ is the cardinal series for $F$.

In particular, we have

H5. The Fourier and the cardinal series coincide for $f \in \mathscr{B}$. They are absolutely convergent in this case.

Finally, we give a slightly weakened form of Hardy's theorem on $T$-cardinal series:

H6. Let $f \in \mathscr{B}$; then the $T$-cardinal series for $f$ converges pointwise to $f$ on $\mathbb{R}$.

1.5. Introduction of the sampling theorem to communication theory. We have already mentioned that the sampling theorem in form (A) was known to Borel in 1897, and both forms (A) and (B) were introduced into the engineering literature by Shannon, whose paper was apparently written in 1940 but not published until after World War II (Shannon (1949)); however, its contents seem to have been in circulation in the United States by 1948 (see p. 10n; also see Shannon (1948)).

Some years later it became known outside Russia that Kotel'nikov (1933) had published the sampling theorem in forms (A) and (B) well before World War II, and, in that country, it had become known by his name. References to Kotel'nikov's work began to appear in western literature in the late 1950s (e.g., Kolmogorov (1956) and Kolmogorov and Tichomirov (1960)). In the early 1960s D. P. Petersen (1963) still felt it desirable to draw it to the attention of the engineering community and summarise its main results.

Let us note two other independent introductions of the sampling theorem. One of these is by Someya (1949) and evidently continues a long Japanese 
interest in cardinal series (going back to Ogura (1920)), but I can only report Someya's contribution at second hand, since I have never been able to obtain a copy of his book.

The other continues in the English tradition. J. D. Weston (1949b) exploited Hardy's result $\mathrm{H} 1$ to give

... a general quantitative theory of communication. The fundamental idea is that a coded message can (like the state of a microphysical system in quantum theory) be represented as a vector, or point, in a space of an infinite number of dimensions, and that the process of transmitting a message over an "ideal" signalling system is (like a pure observation in quantum theory) equivalent to a projection of this vector on to a subspace.... A further idea is the provision, in this subspace, of a set of rectangular axes with respect to which the components of a transmitted message are physical magnitudes associated with definite instants of time.... In this way the geometrical point of view is reconciled with that in which transmission is regarded as a process of interpolation.

Weston went on to mention applications to telephone design. See also Weston (1949a).

Other early contributors to the engineering literature were Nyquist, Bennet and Gabor (see, e.g., Shannon (1949, p. 12)), and Raabe (see, e.g., Butzer (1983, p. 186)). An interesting account of the engineering origins of the sampling theorem was given by Lüke (1978).

\section{STORY Two \\ SOME METHODS FOR DERIVING THE CARDINAL AND ALLIED SERIES}

2.1 Foreword. It is appropriate to begin this story with the " $\delta$-method"; Dirac's delta is a particularly convenient tool in the derivation of sampling theorems, and, although not rigorous, the method is one of power and fecundity.

We shall go on to consider what happens if the hypotheses of the sampling theorem are modified in some way. For example, we can ask for information not only from the function to be reconstructed but from its derivative as well; we can alter the "band region" (the set on which $f^{\wedge}$ has compact support); we can perturb, or "jitter", the sampling instants. These modifications, as well as others, have appeared in the literature largely in response to the needs of engineering applications. Of those mentioned, the first two will be discussed in $\$ \$ 2.3$ and 2.4 ; the third, in $\$ 3.1$. To end the present story, we shall see how the cardinal series is closely related to the classical Poisson summation formula.

2.2. The $\delta$-method. The delta distribution enters the picture naturally here, because the nearest thing one can do on paper to imitate the physical process that an engineer would use to actually sample a signal at the instant $t=T$, that is, to subject the signal $f(t)$ to an "instantaneous" pulse at that time, is to form 
$f(t) \delta(t-T)$. Of course, one cannot sample over an infinite time scale; nevertheless, the "sampled version" $f_{s}$ of $f$,

$$
\begin{aligned}
f_{s}(t) & =f(t) \sum \delta(t-n \tau), \quad \tau>0, \\
& =\sum f(n \tau) \delta(t-n \tau),
\end{aligned}
$$

suggests that $f$ itself be represented as

$$
f(t)=\sum f(n \tau) g(t-n \tau),
$$

in which $g$ is to be a "reconstruction" function independent of $f$. In order to find $g$, first note that (14) can be written

$$
f(t)=\int_{\mathbf{R}} f(u) g(t-u) \sum \delta(u-n \tau) d u
$$

Next, this periodic "delta train" is expanded in Fourier series:

$$
\sum \delta(u-n \tau)=\frac{1}{\tau} \sum \exp \frac{-2 \pi i n t}{\tau} .
$$

On rearranging (15) we find a summand consisting of $f(t) \exp (-2 \pi i n t / \tau)$ convolved with $g(t) / \tau$; taking Fourier transforms,

$$
f^{\prime}(x)=\frac{1}{\tau} \sqrt{2 \pi} g(x) \sum f^{\prime}\left(x+\frac{2 n \pi}{\tau}\right) .
$$

On the right we find a phenomenon frequently encountered in the derivation of sampling theorems-spectrum repetition (the phraseology is common in engineering literature, where a function's Fourier transform is usually called its spectrum). One consequence of this is that if $f$ is band-limited to $[-\pi W, \pi W]$, then we must give $\tau$ the value $1 / W$ to obtain the optimum sample spacing, since any smaller value will cause overlapping of the spectra. Another is that, since there is one copy of $f^{\wedge}$ on the left of (16), we shall have to take $\sqrt{2 \pi} g(x) / \tau$ to be a "window" through which we can "see" just one copy on the right. That one corresponding to $n=0$ will do, so $g^{\wedge}(x)$ must be $\chi(-\pi W, \pi W) / \sqrt{2 \pi} W(\chi$, as usual, denotes the characteristic function of the indicated interval). Taking inverse Fourier transforms we get

$$
g(t)=\frac{\sin \pi W t}{\pi W t},
$$

and (14) is the cardinal series.

Note that this method forces out the "right" form for the reconstruction function $g$ (under the assumption of a series representation of the form (14)), and it even determines the Nyquist sampling rate. What does the lack of rigour matter when one has at one's disposal such a thoroughly healthy, rugged, outdoor method of derivation as this?

It is not easy to trace the origins of the $\delta$-method in the literature; Kohlenberg (1953, p. 1432) has given some early references, however, going back to 1947.

Many different kinds of sampling theorems have been derived using this method. Linden (1959) used it to derive the derivative sampling formulae of 
\$2.3, both kinds of bandpass sampling formulae in \$2.4, and others; a multidimensional version of the method was an essential feature of Petersen and Middleton's (1962) derivation of the formula for sampling over a general lattice (see §5.3).

2.3. Derivative sampling. In order to illustrate a practical sampling situation, J. Fogel (1955) has mentioned the example of an airplane pilot's instrument panel, which traditionally consists of dials with pointers giving information about the plane's altitude, attitude, speed, etc. Pilots scan their instruments, obtaining information from any one of them on a roughly periodic basis. It is possible that derivative information could be available to the pilot as well; for example, the altimeter would be noticed to be "unwinding" at an alarming rate if the plane were in a nose dive! It is just conceivable that the acceleration of the pointer could be observed as well; at any rate this little example does point out the general need for a sampling theorem which takes account of samples not only from the function itself but also from its first $r$ derivatives. When just the samples of $f$ (band-limited to $[-\pi W, \pi W]$ ) and $f^{\prime}$ are available, the formula is

$$
f(t)=\sum\left\{f\left(\frac{2 n}{W}\right)+\left(t-\frac{2 n}{W}\right) f^{\prime}\left(\frac{2 n}{W}\right)\right\}\left\{\frac{\sin \pi(W t-2 n) / 2}{\pi(W t-2 n) / 2}\right\}^{2}
$$

and in this form it was first given by Jagerman and Fogel (1956). A scale factor has been included, because this will be a convenient place to mention that both here and in the ordinary sampling theorem the band-limited requirement on $f$ can be removed by allowing $W$ to approach infinity. The Aachen school (see, e.g., Butzer (1983)) has successfully exploited this idea to obtain several interesting approximation theorems. They also seem to have been the first to attach proper convergence criteria to the derivative sampling formulae and to the bandpass formulae of the next section. For example, (17) holds uniformly on $\mathbb{R}$ if $f$ and $f^{\prime}$ are bounded, continuous, and integrable over $\mathbb{R}$, and $f^{\prime}$ is integrable there. This is for band-limited $f$; if this requirement is dropped, the result holds in the limit $W \rightarrow \infty$. See also $\$ 3.2$.

The general reconstruction formula, which uses samples from a function $f$ and its first $r$ derivatives, was first given by Linden and Abramson (1960). It is

$$
f(t)=\sum P_{r}(n, t)\left[\frac{\sin \pi[(W t-(r+1) n) /(r+1)]}{\pi(W t-(r+1) n) /(r+1)}\right]^{r+1},
$$

where each $P_{r}(n, t)$ is a polynomial of degree $r$ whose form is too complicated to reproduce here. These polynomials were erroneously given by Linden and Abramson, but the error was corrected in their 1961 paper, in which implicit formulae are given from which $P_{r}$ can be calculated. We recall that, with $\alpha=(r+1) n / W, P_{1}=f(\alpha)+(t-\alpha) f^{\prime}(\alpha)$; one also calculates, for example,

$$
P_{2}=f(\alpha)+(t-\alpha) f^{\prime}(\alpha)+(t-\alpha)^{2}(\pi W / 6)\left(f(\alpha)+f^{\prime \prime}(\alpha)\right) .
$$

Note that whatever positive integer value $r$ takes, the spacing between sample points is $r+1$ times that in the ordinary sampling theorem, but since we need $r+1$ samples at each point, the usual Nyquist sampling rate is retained. 
2.4. Altering the band region-bandpass functions. Up to now the band region associated with our band-limited functions has been an interval centered at the origin. This is more than just a notational convenience; because the complex conjugate of $f(-x)$ is $\bar{f}^{\gamma}(x)$, we find that if $f$ is real valued, as it usually is for applications, then $|\hat{f}|$ is even, so the compact support of $f^{\wedge}$ is necessarily symmetric with respect to the origin.

It will be useful to have at our disposal a "shifted" cardinal series for functions $f$ band-limited to the interval $(w-\pi W, w+\pi W)$, and it is a trivial matter to obtain formally, as in (1a), the formula

$$
f(t)=\sum f\left(\frac{n}{W}\right) \frac{\sin \pi(W t-n)}{\pi(W t-n)} \exp \left(i w\left(t-\frac{n}{W}\right)\right) .
$$

Note once again that the Nyquist sampling rate is related to the bandwidth exactly as before.

What is not so trivial is to develop a sampling series for functions which not only fail to contain high-frequency components, but also contain no low-frequency ones either; that is, their band region consists of an interval with a concentric interval removed, say

$$
(-w-\pi W,-w+\pi W] \cup(w-\pi W, w+\pi W] .
$$

Such a function is called a bandpass signal in engineering terminology. If, for example, one were dealing with audio signals, speech or music perhaps, this kind of signal would be encountered; examples also occur in radar.

One could bypass the difficulties altogether by ignoring the "hole" in the band region and simply using the ordinary cardinal series for functions band-limited to [ $-w-\pi W, w+\pi W]$, but intuition tells us that this would be inefficient, since we expect that the overall bandwidth, proportional to $W$ alone, should determine the sampling rate as it has before. This does turn out to be the case, as we shall now see in sketching two of the main approaches to bandpass sampling (for a third, see J. L. Brown (1980)).

In the first of these, we concentrate on the right half, say, of the band region; this approach is suggested by Goldman $(1953$, p. 76$)$. One can do this by writing the bandpass function $f$ by the alternative form of Fourier's integral formula (Titchmarsh (1948, p. 119)) -

where

$$
f(t)=\int_{w-\pi W}^{w+\pi W}(a(u) \cos t u+b(u) \sin t u) d u,
$$

$$
f^{\prime}(x)=a(x)-i b(x)
$$

- and then introducing the auxiliary function

$$
\Phi(x)=\int_{w-\pi W}^{w+\pi W}(a(u)-i b(u)) e^{i x u} d u .
$$

Then $\operatorname{Re} \Phi(x)=f(x)$ and $\operatorname{Im} \Phi(x)=-f(x)$, where $f^{\sim}$ denotes the allied integral or Hilbert transform. Now $\Phi(x)$ admits a shifted cardinal series expansion (above), and taking the real part of this we get the desired modification of the cardinal series for the bandpass function $f$ :

$$
f(t)=\sum\left\{f\left(\frac{n}{W}\right) \cos w\left(t-\frac{n}{W}\right)+\tilde{f}\left(\frac{n}{W}\right) \sin w\left(t-\frac{n}{W}\right)\right\} \frac{\sin w(t-n / W)}{w(t-n / W)}
$$


Some notes. First, the overall bandwidth is $B=4 \pi W$, so the sampling rate is still $B / 2 \pi$ points per second. Second, the presence of the Hilbert transform here seems very natural, but this opinion is not universally held; GonzálesVelasco and Sanvicente (1980, p. 135) take just the opposite view! Third, this sampling series, together with that of the previous section, suggests the general problem of reconstructing $f$ from samples taken from $f$ and from $r$ functionals of it. Steps in this direction have been taken by Papoulis (1968) and J. L. Brown (1981).

A second approach to the sampling of bandpass functions involves "second order sampling" and is due to Kohlenberg (1953), who used the $\delta$-method of derivation. Second order sampling means that one employs two sets of sampling points, each equidistantly spaced with the same spacing and with one set shifted from the other by a factor $\kappa$, say. Then the reconstruction series for our bandpass function is

$$
f(t)=\sum\left\{f\left(\frac{n}{W}\right) g\left(t-\frac{n}{W}\right)+f\left(\kappa+\frac{n}{W}\right) g\left(-t+\kappa+\frac{n}{W}\right)\right\},
$$

in which $g$ is a special reconstruction function whose form is too complicated to reproduce here. Note once again the same Nyquist sampling rate as before.

2.5. Poisson's summation formula. It appears that Boas (1972) was the first to exploit the Poisson summation formula for the express purpose of deriving the sampling theorem. The main object of this section is to sketch this method and note that it carries the added bonus of deriving a certain error bound in the process. Before doing this, let us turn aside for a moment to look at some interesting summation formulae.

First, we note that if it were permissible to integrate the cardinal series representation

$$
f(t)=\sum f(n) \frac{\sin \pi(t-n)}{\pi(t-n)}=\sum f(n) w_{n}(t)
$$

term by term over $\mathbb{R}$, we would at once obtain, using the well-known fact that the integral of each $w_{n}(t)$ has the value unity,

$$
\int_{\mathbf{R}} f(t) d t=\sum f(n) .
$$

This rather striking formula cannot make sense for every $f$ with a cardinal series representation, however. Indeed, we can find a Paley-Wiener function for which the series diverges; take, for example, a sequence $\left(c_{n}\right)$ of reals such that $\sum c_{n}^{2}$ converges, but $\sum c_{n}$ does not, and invoke the Riesz-Fischer theorem in the Hilbert space PW. This, together with the convergence facts in, and following, H1 (\$1.4), gives a Paley-Wiener function with the stated behaviour.

On the other hand, (18) does hold for $f$ band-limited to $[-\pi, \pi]$ and belonging to $L(\mathbb{R})$, since then $f^{\wedge}$ is continuous over $\mathbb{R}$, null outside $(-\pi, \pi)$, and hence belongs to $L^{2}(\mathbb{R})$; thus, $f$ also belongs to $L^{2}(\mathbb{R})$ and, hence, to PW. The cardinal series for $f$ converges uniformly over $\mathbb{R}$ as in Kluvanek's theorem (§4.1), term by term integration is justified, and (18) holds. 
Boas (1972) deduced this result from a general form of Poisson's formula,

$$
\sqrt{\gamma} e^{\pi i \alpha \beta} \sum e^{2 \pi i n \alpha} f(\gamma(n+\beta))=\sqrt{\frac{2 \pi}{\gamma}} e^{-\pi i \alpha \beta} \sum e^{2 \pi i n \beta} f^{\prime}\left(\frac{2 \pi(n-\alpha)}{\gamma}\right),
$$

which, he reports, Hardy used to give in lectures, by taking $\gamma=1, \alpha=\beta=0$.

Formula (18) belongs to the "continuous analogues of series" circle of ideas (Boas and Pollard (1973)). It is also of interest in that it is an exact quadrature formula. The idea goes back to Wiener (see Bhatia and Krishnan (1948, p. $184 \mathrm{n})$ ), who used it to show that

$$
\sum\left[\frac{\sin (n \alpha+\theta)}{n \alpha+\theta}\right]^{2}=\frac{\pi}{\alpha} .
$$

Boas also deduced the summation formula

$$
\sum(-1)^{n} f(n)=0
$$

under the same hypotheses on $f$ by taking $\gamma=2, \alpha=0, \beta=1 / 2$ in Poisson's formula. Note that, for essentially the same reason, (19) does not generalise to PW any more than (18) did. Interestingly enough, however, the scaled version

$$
\sum(-1)^{n} f(n \tau)=0, \quad 0<\tau<1,
$$

does hold for all $f \in \mathrm{PW}$ (see Zakai (1965, p. 148) for this and another criterion for (19) to hold).

Turning now to the derivation of the cardinal series, we note first that the absolute error incurred in expanding an arbitrary function in cardinal series, $\left|f(t)-\sum f(n) w_{n}(t)\right|$, is called the aliasing error and is important for applications, because one may wish to sample a function whose bandwidth, even though finite, may not be known a priori.

We shall now need Poisson's formula in the form

$$
\sqrt{2 \pi} \sum f^{\prime}(2 n \pi-t)=\sum f(n) e^{i n t},
$$

virtually as it was given (without proof) by Gauss $(1900$, p. 88 ) in a note written sometime between 1799 and 1813 .

After our previous discussions, this formula is redolent of possibilities; on the left we recognise spectrum repetition, while the series on the right has all the appearance of a Fourier series, which, if multiplied by $e^{-i x t}$ and integrated from $-\pi$ to $\pi$, would yield the cardinal series.

Now suppose that

$$
f(t)=\frac{1}{\sqrt{2 \pi}} \int_{\mathbf{R}} g(u) e^{i u t} d u, \quad g \in L(\mathbb{R}) .
$$

Let us formally replace $f$ in (20) with $g$ and carry out the integration procedure just mentioned. We can then write the aliasing error as

$$
\frac{1}{\sqrt{2 \pi}}\left|\sum\left(1-e^{-2 \pi i n t}\right) \int_{(2 n-1) \pi}^{(2 n+1) \pi} g(u) e^{i u t} d u\right| \text {. }
$$

The term corresponding to $n=0$ vanishes, and, after an obvious estimate and reconstitution of the integral, we find that this aliasing error is bounded by

$$
B=\frac{2}{\sqrt{2 \pi}} \int_{|u|>\pi}|g(u)| d u \text {. }
$$


This result goes back to P. Weiss; see J. L. Brown (1967), who derived it (by a different method than that just given) for $f$ as in (21), together with a similar bound for bandpass functions.

A nice little example by Brown shows that the constant $2 / \sqrt{2 \pi}$ cannot be improved. Take $f(t)=[\sin 2 \pi(t-1 / 2)] / \pi(t-1 / 2)$, which vanishes at every integer sampling point. Hence, the aliasing error cannot exceed $\max |f(t)|=2$; but the value of $B$ for this $f$, calculated as above, is also 2 .

For many other results on the aliasing error see Jerri (1977, §VI B).

Returning to the Poisson summation method, we see that the cardinal series representation for $f$ is obtained formally if $g$ in (21) vanishes outside $[-\pi, \pi]$. See Butzer (1983, p. 188) for the detailed derivation, which additionally requires that $f \in L(\mathbb{R})$.

Boas remarked that the same results can be obtained from the Euler-Maclaurin summation formula. Earlier, Kohlenberg (1953) had also pointed out the relevance of the Euler-Maclaurin formula; the details have been given by Butzer and Stens (1983).

\section{STORY THREE \\ $L^{2}$ AND $L^{p}$ THEORY}

3.1. Bases for the Hilbert space of Paley-Wiener functions. Hardy's results $\mathrm{H} 1$ and $\mathrm{H} 2$ (\$1.4) form the core of the $L^{2}$ theory of cardinal series. We shall expand a little on these results; in this section we look at several interesting bases for PW, all of which are obtainable by Hardy's method; in the next section we look at a generalisation of the reproducing equation, as well as some results about operators on $\mathrm{PW}$.

We denote by $\mathscr{L}^{2}(-\pi, \pi)$ that subspace of $L^{2}(\mathbb{R})$ whose members are null outside $\left[-\pi, \pi\right.$ ]; clearly $\mathscr{L}^{2}(-\pi, \pi)$ is isometrically isomorphic to $L^{2}(-\pi, \pi)$. Hardy used the Plancherel theory to show that a CON set in $\mathscr{L}^{2}(-\pi, \pi)$ maps, under $\mathscr{F}^{-1}$, the inverse Fourier transform, to a CON set in PW; note that this mapping also preserves bases, even Riesz bases (see, e.g., Young (1980, p. 30)). Table 1 contains a list of some of the possibilities, and some comments follow.

TABLE 1

\begin{tabular}{lll} 
& \multicolumn{1}{c}{$\mathscr{L}^{2}(-\pi, \pi)$} & \multicolumn{1}{c}{$\mathrm{PW}$} \\
\hline \hline 1. & $e^{-\ln x} / \sqrt{2 \pi} \quad(n \in \mathbf{Z})$ & $w_{n}(t)$ \\
2. & $\operatorname{sgn} x e^{-\ln x} / \sqrt{2 \pi} \quad(n \in \mathbf{Z})$ & {$[\pi(t-n) / 2]^{-1} \sin ^{2} \pi(t-n) / 2$} \\
3a. $e^{-i \lambda_{n} x} / \sqrt{2 \pi}$, & $w_{\lambda_{n}}(t)$ \\
& $\left|\lambda_{n}-n\right| \leqslant D<\frac{1}{4} \quad(n \in \mathbf{Z})$ & $H(t) / H^{\prime}(t)\left(t-\lambda_{n}\right)$, \\
3b. $h_{n}(x)$ & $H(t)=\left(t-\lambda_{0}\right) \prod_{n-1}^{\infty}\left(1-t / \lambda_{n}\right)\left(1-t / \lambda_{-n}\right)$ \\
& & $\left(n+\frac{1}{2}\right)^{1 / 2}(-1)^{n}(t \pi)^{-1 / 2} J_{n+1 / 2}(t \pi)$ \\
4. $\quad\left(n+\frac{1}{2}\right)^{1 / 2} P_{n}(x / \pi), \quad n=0,1, \ldots$. & $(-i)^{n}\left(2 \pi v_{n} / \sigma\right)^{1 / 2} \phi_{n}(t / \sigma), \quad \sigma>0$ \\
5. $\quad \phi_{n}(x) / \sqrt{\nu_{n}}$ &
\end{tabular}




\section{Comments.}

Item 1 . Needs no further comment.

Item 2. That $\left\{\operatorname{sgn} x e^{i n x} / \sqrt{2 \pi}\right\}$ is $\operatorname{CON}$ in $\mathscr{L}^{2}(-\pi, \pi)$ follows from multiplying the trigonometrical set of Item 1 by a function whose modulus is one a.e.; this alters neither its completeness nor its orthonormality. It does not seem to have been pointed out before that the corresponding set in $\mathrm{PW}$ is in fact CON there. The expansion in this set for $f \in \mathrm{PW}$ is

$$
f(t)=\sum-f(n) \frac{\sin ^{2} \pi(t-n) / 2}{\pi(t-n) / 2},
$$

the Hilbert transform entering the form of the coefficients as in Titchmarsh (1948, p. 120). This expansion formula was previously known for a more restrictive class of functions (Butzer and Splettstösser (1977, p. 47)).

Item $3 \mathrm{a}$. The function set in the first column is a Riesz basis for $\mathscr{L}^{2}(-\pi, \pi)$, which is in many ways the next best thing we can ask for after a CON set. This is a result of Kadec; see Young (1980) and Higgins (1977) for this and many other similar criteria. We see that the set $\left\{w_{\lambda_{n}}\right\}$ in the second column forms a Riesz basis for PW. The sample points $\left(\lambda_{n}\right)$ are perturbations (not too large) of the integers; perturbations of this kind are sometimes called "jitter" in engineering terminology.

Item $3 \mathrm{~b}$. Since the biorthogonal set to a basis for a Hilbert space is also a basis, we consider here the biorthogonal sets for those in $3 \mathrm{a}$ (we do not need the precise form of the functions $\left(h_{n}\right)$ ). For references to the form of the function set in the second column, see, e.g., Higgins (1976). We see that, under a perturbation of the sample points, the cardinal series splits into two different representations for a PW function; the first is not an interpolation series, but its expansion functions are perturbations of the ordinary ones, while the second is clearly an interpolation series of Lagrange type. For much more on jitter, which in applications is often stochastic in nature, see Jerri (1977, §§IVD and VIC).

Item 4. The Legendre polynomials form a CON set in $\mathscr{L}^{2}(-\pi, \pi)$; their inverse Fourier transforms in the second column form a set of functions associated with the classical Bessel-Neumann series. Its completeness in PW was pointed out by Higgins (1972, p. 712).

Item 5. It follows from the standard Hilbert-Schmidt theory of integral operators with symmetric kernel that the operator $\mathscr{P}$, where

$$
\mathscr{P}_{\phi}(t)=\int_{-\pi}^{\pi} \phi(x) \frac{\sin \sigma(t-x)}{\pi(t-x)} d x, \quad \phi \in L^{2}(-\pi, \pi),
$$

has infinitely many eigenvalues $\left(\nu_{n}\right)$ with corresponding eigenfunctions $\left(\phi_{n}\right)$ which form a CON set in $\mathscr{L}^{2}(-\pi, \pi)$. These are the prolate spheroidal wave functions and they have several remarkable properties, not the least of which is the Fourier transform property indicated in Item 5. The set in the second column is a CON set for PW and has been studied intensively by Landau, Pollack, and Slepian. One of their results is that the expansion for a 
Paley-Wiener function in prolate spheroidal functions is superior to the ordinary cardinal series with respect to certain truncation errors. See, e.g., Landau and Pollack (1962) and also Landau (1967a, p. 1703).

3.2. Further results for $P W$. the equality between convolution integral and convolution sum in the following theorem is one way of generalising the reproducing equation of $\mathrm{H} 2, \$ 1.4$.

THEOREM. If $f$ and $g$ belong to $\mathrm{PW}$ then

$$
\int_{\mathbf{R}} f(u) g(t-u) d u=\sum f(n) g(t-n)
$$

is the series converging uniformly in t on compact subsets of $\mathbb{R}$.

Of course, (22) is nothing more than a form of Parseval's relation in PW (cf. Stens (1980, p. 40)); the result follows in a standard way from an $L^{2}$ convolution theorem (Titchmarsh (1948, p. 90)), which states that if $f$ and $g$ belong to $L^{2}(\mathbb{R})$ then the left side of $(22)$ equals

$$
\int_{\mathbf{R}} f^{(}(x) g^{\Upsilon}(x) e^{-i t x} d x \text {. }
$$

We shall look at two special cases.

First, take $g(t)=(\pi t / 2)^{-1} \sin ^{2} \pi t / 2$. On using (22a) the left side of (22) becomes $-i\left(f^{\top}(x) \operatorname{sgn} x\right)^{\gamma}(t)$, and we recognise the signum rule for the Hilbert transform $f^{\sim}$ of $f$. Thus, for every $f$ belonging to $\mathrm{PW}$,

$$
f(t)=\sum f(n) \frac{\sin ^{2} \pi(t-n) / 2}{\pi(t-n) / 2},
$$

an expansion which bears an obvious relation of duality to that in Item 2 of the previous section. Actually, using $\tilde{f^{\sim}}=-f$ a.e., $f \in L^{2}(\mathbb{R})$, either result can be obtained from the other.

Our second special case gives the derivative sampling formula which we met in (17) of §2.3. Let us start with $f \in \mathrm{PW}$ so that

$$
f(-t)=\frac{1}{\sqrt{2 \pi}} \int_{-\pi}^{\pi} f^{\mathcal{Y}}(u) e^{-i u t} d u=\frac{1}{\sqrt{2 \pi}} \int_{-k \pi}^{k \pi} F^{\mathcal{Y}}(x) e^{-i x \tau} d x,
$$

on putting $x=k u, \tau=t / k$, and $F(x)=f(k x), k>0$. Clearly the factor $(x \operatorname{sgn} x) / k \pi+(1-|x| / k \pi)$ can be inserted into the integrand, and the integral can be rearranged in two parts; (22a) is then applied to each part in order to assemble the left side of (22). From this, the formula

$$
f(t)=\frac{k}{2} \sum\left\{F^{\prime}(n)(\tau-n)+F(n)\right\} S_{k}(\tau)
$$

can be obtained, where $S_{k}(\tau)$ denotes $(k \pi \tau / 2)^{-2} \sin ^{2} k \pi \tau / 2$. However, care must be taken with (22), since its application is only valid if $S_{k}(\tau) \in \mathrm{PW}$, which will only be the case if $k \leqslant 2$. This restriction is only to be expected on intuitive grounds, since $k>2$ would give a sampling rate less than Nyquist.

The cases $k=1,2$ give, for every $f \in \mathrm{PW}$,

$$
f(t)=\frac{1}{2} \sum\left\{f^{\prime}(n)(t-n)+f(n)\right\}\left[\frac{\sin \pi(t-n) / 2}{\pi(t-n) / 2}\right]^{2}
$$


and

$$
f(t)=\sum\left\{f^{\prime}(2 n)(t-2 n)+f(2 n)\right\}\left[\frac{\sin \pi(t-2 n) / 2}{\pi(t-n) / 2}\right]^{2},
$$

respectively. These two formulae are noted by Butzer and Splettstösser (1977) under more restrictive hypotheses. A direct comparison of the two shows that the " $\frac{1}{2}$ " in the first one can be interpreted by taking the sum in two parts-one where the summation is over the even integer sampling points; the other where it is over the odd integer sampling points-each part representing $f$. Once again, this is only to be expected on intuitive grounds.

I would like to end this section by mentioning some results of Weston (1949) on operators on PW, which we sum up in the theorem below. First let the dilation operator $\delta_{a}$ be defined, for real nonzero $a$, by

$$
\delta_{a}: f(t) \rightarrow|a|^{1 / 2} f(a t) .
$$

Then $\delta_{a}$ is a unitary operator on $L^{2}(\mathbb{R})$.

Let the translation operator $\tau_{k}$ be defined, for real $k$, by

$$
\tau_{k}: f(t) \rightarrow f(t-k) \text {. }
$$

Then $\left\{\tau_{k}\right\}$ is a one-parameter continuous group of unitary operators on $L^{2}(\mathbb{R})$.

Further, let $D$ and * denote differentiation and convolution as usual.

THEOREM. 1. If $|a| \leqslant 1$, PW is an invariant subspace for $\delta_{a}$. If $|a| \geqslant 1, \mathrm{PW}^{\perp}$ is invariant.

2. $\mathrm{PW}$ and $\mathrm{PW}^{\perp}$ are invariant subspaces for $\tau_{k}$.

3. $-i D$ is a bounded selfadjoint operator on PW. Its spectrum is the continuum $[-\pi, \pi]$.

4. Let $f \in L^{2}(\mathbb{R}), g \in \mathrm{PW}$, and $f * g \in L^{2}(\mathbb{R})$. Then $f * g \in \mathrm{PW}$.

To these results let us add

5. $\mathrm{PW}$ and $\mathrm{PW}^{\perp}$ are invariant subspaces for Hilbert transformation on $L^{2}(\mathbb{R})$.

REMARKS. In 1 of the theorem we note that scaling by a factor $a,|a|<1$, will reduce the bandwidth, and it might be preferable to regard $f(a t)$ as belonging to a different PW space.

It is clear by considering the convolution properties of the Fourier transform that the product of $k$ members of $\mathrm{PW}$ will be band-limited, but with $k$ times the original bandwidth; however, if each of these $k$ members are scaled by a factor $1 / k$, their product will belong to $\mathrm{PW}$. This seems to cover certain remarks of Weston (1949a, particularly the bottom of p. 339ff.) about members of the basis $\left\{w_{n}\right\}$ for PW; these remarks must be treated with some circumspection since they are based on formulae which appear to be incorrect as they stand.

For other discussions of operators on PW see Kramer (1973) and Mugler (1976). It should be pointed out that much of the theory of operators on PW is but a special case of the more general theory on Hilbert spaces with reproducing kernel, a point that we shall touch on at the end of the next section. 
3.3. Beyond the Fourier transform. It was J. M. Whittaker (1935, p. 71) who first pointed out that kernels other than the Fourier kernel can be used as a basis for a cardinal type series, mentioning explicitly the Hankel kernel $(x t)^{1 / 2} J_{0}(x t)$. Like the Fourier kernel, which yields a complete orthogonal set in $L^{2}(-\pi, \pi)$ when integer values are given to one of its arguments, the Hankel kernel yields a complete orthogonal set in $L^{2}(0,1)$ when one argument is evaluated at the zeros of $J_{0}$. It is just this idea of using an integral operator whose kernel $K(t, u)$ has an associated sequence $\left(t_{n}\right)$, such that $\left\{K\left(t_{n}, u\right)\right\}$ is a complete orthogonal set in some $L^{2}(a, b)$ space, that is the chief ingredient of Kramer's generalisation of the cardinal series. The idea had also been suggested by Weiss, but Kramer (1957) gave the following

THEOREM. Let

$$
f(t)=(\mathscr{K} g)(t)=\int_{a}^{b} K(t, u) g(u) d u,
$$

where $g \in L^{2}(a, b), K(t, u) \in L^{2}(a, b)$ for each $t \in \mathbb{R}$, and $\left\{K\left(t_{n}, u\right)\right\}$ forms $a$ complete orthogonal set in $L^{2}(a, b)$ for some $\left(t_{n}\right)$. Then

$$
f(t)=\sum f\left(t_{n}\right) s_{n}(t),
$$

where $s_{n}(t)=\left\langle K(t, \cdot), K\left(t_{n}, \cdot\right)\right\rangle /\left\|K\left(t_{n}, \cdot\right)\right\|^{2}$.

Here, $\langle\cdot, \cdot\rangle$ denotes the inner product and \|\| denotes the norm.

However, much more needs to be assumed about $\mathscr{K}$ if we are to parallel the r.k. setup which holds in the ordinary Fourier case. There, an essential feature is the unitary character of the Fourier transform on $L^{2}(\mathbb{R})$; also the r.k. is the inner product of the Fourier kernel with itself.

Conditions sufficient for some of the r.k. theory to hold in this more general setting have been given by Higgins (1972). Thus, if $\mathscr{K}: L^{2}(a, b) \rightarrow L^{2}(\mathbb{R})$ is bounded and has a bounded inverse, then its range does have r.k., and, further, if $\mathscr{K}$ is unitary this r.k. is indeed given by the inner product of the kernel $K$ with itself. The special case of the Hankel kernel $(x t)^{1 / 2} J_{\nu}(x t), \nu>-1$, has been studied as a case in which it is possible to meet the rather lengthy list of requirements on $\mathscr{K}$.

Indeed, if $\left\{j_{\nu n}, n=0,1, \ldots\right\}$ denotes the set of positive zeros of the Bessel function $J_{\nu}(t), \nu>-1$, then the class of functions $\{f\}$ belonging to $L^{2}(0, \infty)$, whose Hankel transform

$$
\left(\mathscr{H}_{\nu} f\right)(t)=\underset{A \rightarrow \infty}{\operatorname{lim.m}} \int_{0}^{A}(x t)^{1 / 2} J_{\nu}(x t) f(x) d x
$$

is null outside $[0,1]$, is a Hilbert space whose reproducing kernel, when one of its arguments is evaluated at $j_{\nu n}$, yields the CON set

$$
s_{n}(t)=\frac{2\left(j_{\nu n} t\right)^{1 / 2} J_{\nu}(t)}{J_{\nu}^{\prime}\left(j_{\nu n}\right)\left(t^{2}-j_{\nu n}^{2}\right)}, \quad n=0,1, \ldots
$$

The expansion for such an $f$ is then

$$
f(t)=\sum_{n=0}^{\infty} f\left(j_{\nu n}\right) s_{n}(t)
$$


See Jerri (1977, §IIIA) for further references to this kind of cardinal series. Special expansions for Bessel functions can be obtained as particular cases in just the same way that the ordinary cardinal series can be made to yield special expansion formulae, a large collection of which can be found in Magnus et al. (1966, pp. 136, 180). See also Jerri (1977, p. 1592).

Both Weiss and Kramer had pointed out that kernels of the type required for Kramer's theorem to hold can be obtained formally in association with eigenfunctions of selfadjoint differential operators, and many examples of this have appeared in the literature. See Jerri (1977, §§IIIA, F); see also Mehta (1975) for additional references. It must be said, however, that these tend to be rather ad hoc collections of formulae, and, while operational properties of many of the kernels involved are in the literature, these examples have not been integrated into a theoretical framework like that described above.

The connection with differential operators is not essential; in fact, Kak (1970) has derived the Walsh sampling theorem (see $\$ 4.2$ ) as a special case of Kramer's theorem.

A "bandpass" version of the Kramer theorem has been given by Sharma and Mehta (see, e.g., Jerri (1977, §IVE-1)).

The presence of a reproducing kernel in $H$, the range of the operator $\mathscr{K}$, suggests that the action of operators on $H$ is likely to be closely connected with this kernel and, hence, with the sampling expansion.

THEOREM. Let $H$ be a separable Hilbert function space with reproducing kernel $k(u, t), u, t \in \mathbb{R}$, and let $\left(t_{n}\right), n \in \mathbb{Z}$, be a sequence of reals such that $\left\{k\left(u, t_{n}\right)\right\}$ is a complete orthogonal set in $H$. Let $\mathscr{T}$ be a bounded linear operator on $H$ with adjoint $\mathscr{T}^{*}$. Then for each $f \in H$,

$$
\begin{aligned}
\mathscr{T} f(t) & =\sum f\left(t_{n}\right) \mathscr{T} k\left(\cdot, t_{n}\right)(t) \\
& =\sum \mathscr{T} f\left(t_{n}\right) k\left(t, t_{n}\right) \\
& =\left\langle f, \mathscr{T}^{*} k(\cdot, t)\right\rangle .
\end{aligned}
$$

These results are not deep. The second is obvious, and the third follows at once from the reproducing kernel theory (Meschowski (1962, p. 50)). As to the first, we have

$$
\left\|\mathscr{T} f-\sum^{N} f\left(t_{n}\right) \mathscr{T} k\left(\cdot, t_{n}\right)\right\| \leqslant\|\mathscr{T}\|\left\|f-\sum^{N} f\left(t_{n}\right) k\left(\cdot, t_{n}\right)\right\|,
$$

but strong convergence implies pointwise convergence in a Hilbert space with reproducing kernel, hence the result.

For example, if $H$ is $\mathrm{PW}$ and $\mathscr{T}$ is the Hilbert transform, then one finds, on using a special transform, that the first and third results are just those that we found in $\$ 3.2$ as the first special case of (22).

3.4. Absolute convergence. R. P. Gosselin (1963) says that "the convergence behavior of cardinal series is, in general, quite favorable...". Let us begin this section by looking at some of the classical convergence theorems. 
Theorem 1 (Uniform Convergence Principle). If a cardinal series converges for a single noninteger value of $t$, it converges uniformly on compact subsets of the complex t-plane to an entire function of $t$.

Theorem 2 (Absolute Convergence Principle). A cardinal series $\sum a_{n} w_{n}(t)$ converges absolutely if and only if

$$
\sum^{\prime}\left|\frac{a_{n}}{n}\right|<\infty
$$

Both of these theorems appear in the work of J. M. Whittaker (1929a, p. 42; $1929 \mathrm{~b}$, p. 171, resp.). The first is really part of a more comprehensive result, being true if $(C, k)$ summability is inserted instead of convergence. We also have

THEOREM 3. Let

$$
f(t)=\int_{-\pi}^{\pi} g(u) e^{i t u} d u, \quad g \in L^{1}(-\pi, \pi) .
$$

Then $f$ is represented by its cardinal series pointwise on $\mathbb{R}$.

It now seems natural to adopt the following

Definition. Let us say that $f \in \mathrm{BL} p$ if

$$
f(t)=\int_{-\pi}^{\pi} g(u) e^{i t u} d u, \quad g \in L^{p}(-\pi, \pi) .
$$

Note that $\mathrm{BL} 2 \equiv \mathrm{PW}$, and $\mathrm{BL} p \subset \mathrm{BL} 1, p>1$.

In order to approach Theorem 4 we need a further definition and subsequent inequality.

Definition. We say that $g$ belongs to $\operatorname{Re} H^{1}$ if $g$ consists of the boundary values of the real part of a function $h$ belonging to the Hardy space $H^{1}$.

Hardy's inequality. If $\left(c_{n}\right)$ are the Fourier coefficients of $g \in \operatorname{Re} H^{1}$ then $\sum\left|c_{n} / n\right|<\infty$.

It is well known that $L^{p}(-\pi, \pi) \subset \operatorname{Re} H^{1} \subset L^{1}(-\pi, \pi), p>1$.

THEOREM 4. (i) If $f \in \mathrm{BL} p, p \geqslant 1$, then it is represented by its cardinal series pointwise on $\mathbb{R}$, hence uniformly on compact subsets.

(ii) The convergence is absolute if $p>1$. When $p=1$ the convergence may fail to be absolute, but it is absolute if $g$ in (26) belongs to $\operatorname{Re} H^{1}$.

Part (i) is a consequence of the facts assembled in this section. As to part (ii), note first that when $p>1$ the absolute convergence follows from Hölder's inequality, since the Fourier coefficients for $g$ in (26), which are $\{f(n) / \sqrt{2 \pi}\}$, belong to $l^{q}, p+q=p q$.

The interesting part is what happens when $p=1$. Now if $g \in \operatorname{Re} H^{1}$, we find that $a_{n}$ in the absolute convergence criterion (25) and $c_{n}$ of Hardy's inequality are both equal to $f(n) / \sqrt{2 \pi}$, so that the two criteria are one and the same. It only remains to show, therefore, that absolute convergence may fail if $g \notin \operatorname{Re} H^{1}$. A classical example from the theory of Fourier series shows this; 
indeed, let

$$
g(u)=\sum_{n=2}^{\infty} \frac{\cos n u}{\log n} .
$$

This $g$ belongs to $L^{1}(-\pi, \pi)$, and the series is its Fourier series; but Hardy's inequality and (25) both fail, and $g \notin \operatorname{Re} H^{1}$.

A phenomenon of Fourier analysis, which has often been remarked on (see, e.g., Coifman and Weiss (1977, particularly p. 271ff.)), is that there are many results about $L^{p}(-\pi, \pi), p>1$, which do not carry over to all of $L^{1}(-\pi, \pi)$ but do hold for $\operatorname{Re} H^{1}$. In view of the equivalence of Hardy's inequality and the absolute convergence criterion for cardinal series when $g \in \operatorname{Re} H^{1}$, we find a very natural occurrence of this phenomenon in the second half of Theorem 4.

3.5. Cardinal series and distributions. This material does not properly belong to $L^{p}$ theory, but follows on rather naturally from it.

Let us first consider one of the representations for $\mathrm{Si}(t)$, the sine integral function:

$$
\mathrm{Si}(t)=\frac{1}{2 i} \mathrm{P} . \mathrm{V} \cdot \int_{-1}^{1} \frac{e^{i u t}}{u} d u .
$$

Zakai $(1965$, p. 143) has pointed out that $\mathrm{Si}(t)$ occurs naturally in applications in that it represents the response of an ideal low-pass filter to a step function. However, it does not belong to any of our BL $p$ classes, since the integral is the transform of a principal value distribution. $\mathrm{Si}(t)$ does suggest, though, that we consider the possibility of expanding into cardinal series the Fourier transform of a distribution with bounded support.

Let us look at another

EXAMPLE. The cardinal series for $e^{i a t}, t \in \mathbb{R}, a \in(-\pi, \pi)$, regarded as a function of $a$, is $\sum e^{i a n} w_{n}(t)$. This is also the Fourier series for $e^{i a t}$ regarded as a function of $a$. It converges to $e^{i a t}, a \in(-\pi, \pi)$, uniformly in $t$ on compact subsets of $\mathbb{R}$.

Note that this cardinal series does not converge absolutely. The representation

$$
e^{i a t}=\int_{-\pi}^{\pi} e^{i u t} \delta(u-a) d u
$$

is another kind of substitute for the usual band-limited property. The example was given by Hardy $(1941$, p. 334$)$ and by Campbell $(1968$, p. 626$)$ from two rather different points of view. It shows, for example, that the existence of the sum of a cardinal series is no guarantee that this sum is band-limited in the conventional sense.

Here is another example, also by Campbell (op. cit.).

EXAMPLE. Replace $\delta$ in the previous example by $\delta^{\prime}$. The cardinal series for ite $^{i a t}$ diverges.

Two questions immediately suggest themselves.

First, can we find an appropriate modification of the cardinal series that will represent at least those functions appearing in our examples? It is known that one can introduce a convergence enhancing factor which will do this. 
Second, for what class of Fourier transforms of distributions of bounded support can we expect an ordinary cardinal series representation? Here, it is known that an appropriate class is the dual of $S$, the class of $C^{\infty}$ functions of rapid descent with $f^{\wedge}$ supported on $[-\pi, \pi]$. See Jerri $(1977, \S I V G)$ for further remarks and references to the very small amount of literature there is in this area.

\section{STORY FOUR \\ THE CARDINAL SERIES AND LCA GROUPS}

4.1. The main theorem. A very general form of the sampling theorem was given by Kluvanek (1965) in the setting of harmonic analysis on LCA (locally compact abelian) groups. In this section we describe this theorem, and in the next we look at several examples.

Let $G$ be a LCA group (written additively) with a discrete subgroup $H$. Let $\Gamma$ be the dual of $G$, and let the value of $\gamma \in \Gamma$ at $x \in G$ be denoted by $(x, \gamma)$. Let $\Lambda \subset \Gamma$ be the (discrete) annihilator of $H$, i.e.,

$$
\Lambda=\{\gamma \in \Gamma:(h, \gamma)=1(h \in H)\}
$$

We set the Haar measures in a very standard way. Discrete groups are given counting measure, and compact groups are given total measure one. We denote the Haar measure on a set $S$ by $m_{S}$ and normalise $m_{G}$ for Fourier inversion; thus,

$$
f^{\mathcal{\prime}}(\gamma)=\int_{G}(-x, \gamma) f(x) d m_{G} \text { and } f(x)=\int_{\Gamma}(x, \gamma) f^{\mathcal{Y}}(\gamma) d m_{\Gamma}
$$

provide a unitary equivalence between $L^{2}(G)$ and $L^{2}(\Gamma)$ via the Fourier-Plancherel theory. Finally, Haar measure on $\Gamma$ is normalised so that a standard integration formula shall hold; in the present context this is

$$
\int_{\Gamma} f(\gamma) d m_{\Gamma}=\int_{\Gamma / \Lambda} \sum_{\lambda \in \Lambda} f(\gamma+\lambda) d m_{\Gamma / \Lambda}
$$

Next, let $\Omega$ be a measurable subset of $\Gamma$ such that for each $\gamma \in \Gamma, \Omega \cap$ $(\gamma+\Lambda)$ consists of a single point. It is standard to take $\Omega=\Gamma / \Lambda$, but certain other choices can also lead to interesting examples.

To complete these definitions let us call

$$
\phi(x)=\int_{\Omega}(x, \gamma) d m_{\Gamma}
$$

the reconstruction function, since one easily shows that it has the interpolatory property

$$
\phi(x)=0, \quad x \in H, x \neq 0, \quad \phi(0)=1
$$


THEOREM (KLUVANEK). Let $f \in L^{2}(G)$ and let $f^{\wedge}$ be null outside $\Omega$. Then $f$ is almost everywhere equal to a continuous function; if $f$ itself is continuous, then its cardinal series expansion is

$$
f(x)=\sum_{y \in H} f(y) \phi(x-y),
$$

the convergence being both uniform on $G$ and in the norm of $L^{2}(G)$. Also

$$
\|f\|^{2}=\sum_{y \in H}|f(y)|^{2} .
$$

\subsection{Examples of cardinal series on $\mathbb{R}, T, \mathbb{T}^{2}, \mathbb{Z}, \Delta$, and $\mathbb{R}^{N}$.}

EXAMPLE 1(a). Let us take $G$, and hence $\Gamma$, to be $\mathbb{R}$. Let $H=(1 / W) \mathbb{Z}$, $W>0$. Then $\Omega=\Gamma / \Lambda=(-\pi W, \pi W]$, and the scaled version (4) of the cardinal series results.

EXAMPLE 1(b). Let $G, \Gamma$, and $H$ be as above, but let $\Omega=(-A-\pi W,-A$ ] $\cup(A, A+\pi W]$ as for the bandpass sampling of $\$ 2.4$. Now the condition that $\Omega \cap(\gamma+\Lambda)$ be a single point for each $\gamma \in \Gamma$ is equivalent to requiring that the points of $\Omega$ be pairwise incongruent $\bmod 2 \pi W$, and this in turn means that $A$ must be a positive integer multiple of $\pi W$, say $\pi W s$. Hence,

$$
\Omega=(-\pi W(s+1),-\pi W s] \cup(\pi W s, \pi W(s+1)] .
$$

Also

$$
\phi(t)=\frac{1}{2 \pi W} \int_{\Omega} e^{i t u} d u=\frac{\sin \pi W(s+1) t-\sin \pi W s t}{\pi W t} .
$$

Thus, from our theorem $(\S 4.1)$ we find that if $f \in L^{2}(\mathbb{R})$ and $\hat{f}$ is null outside $\Omega$, then

$$
f(t)=\sum f\left(\frac{n}{W}\right) \frac{\sin (s+1) \pi(W t-n)-\sin s \pi(W t-n)}{\pi(W t-n)}
$$

with the appropriate modes of convergence.

This series is one that had been given by Kohlenberg (1953), without any convergence criteria, in the context of bandpass sampling.

EXAMPLE 2. Let $G=\mathbb{T}$, the "circle group" consisting of complex numbers of modulus one under multiplication. Let $H$ be the finite subgroup generatecd by $\kappa$, a primitive $k$ th root of unity. We have

$$
\Gamma=\left\{z^{n}, n \in \mathbb{Z}\right\}, \quad \Lambda=\left\{z^{k n}, n \in \mathbb{Z}\right\} .
$$

Take $\Omega=\Gamma / \Lambda=\left\{z^{j}, j=0, \ldots, k-1\right\}$. Then

$$
\phi(z)=\frac{1}{k} \sum_{n=0}^{k-1} z^{n}=\frac{1-z^{k}}{k(1-z)},
$$

and

$$
f^{\mathcal{Y}}(n)=\frac{1}{2 \pi} \int_{0}^{2 \pi} e^{-i n \theta} f\left(e^{i \theta}\right) d \theta
$$


where $f(z) \in L^{2}(C), C$ denoting the unit circle. If $f^{\wedge}$ is null outside $\Omega$, we have, changing the notation slightly,

$$
\int_{C} z^{-n-1} f(z) d z=0, \quad n=k, k+1, \ldots
$$

We can now write a remarkable finite cardinal series representation for functions satisfying the conditions above, which was given by Cauchy in 1841 (p. 286):

$$
f(z)=\frac{1}{k} \sum_{n=0}^{k-1} f\left(\kappa^{n}\right) \frac{\kappa^{n}\left(1-z^{k}\right)}{\kappa^{n}-z} .
$$

EXAMPLE 3. Let $G=\pi^{2}$ with subgroup $H$ generated by $\kappa$ and $\lambda$, primitive $k$ th and $l$ th roots of unity, respectively. Then we get the following two-dimensional finite cardinal series, also due to Cauchy (p. 290):

$$
f(z, w)=\frac{1}{k l} \sum_{n=0}^{k-1} \sum_{m=0}^{l-1} f\left(\kappa^{n}, \lambda^{m}\right) \frac{\kappa^{n}\left(1-z^{k}\right)}{\kappa^{n}-z} \frac{\lambda^{m}\left(1-z^{k}\right)}{\lambda^{m}-z}
$$

for functions $f \in L^{2}(C \times C)$ such that

$\int_{C \times C} z^{-r-1} z^{-s-1} f(z, w) d z d w=0, \quad r=k, k+1, \ldots$ and $s=l, l+1, \ldots$

Cauchy's originals for these two series contained scale factors, which would have entered here if we had taken our circles to be of nonunit radius.

EXAMPLE 4. Let $G$ be $\mathbb{Z}$, with subgroup $H=k \mathbb{Z}, k$ a fixed integer greater than one. We have $\Gamma=\left\{e^{i n y}, 0 \leqslant y<2 \pi\right\}$ and $\Lambda=\left\{e^{2 \pi i j n / k}, j=0,1, \ldots\right.$, $k-1\}$. Take $\Omega=\Gamma / \Lambda=[0,2 \pi / k)$ and then

$$
\phi(n)=\frac{k}{2 \pi} \int_{0}^{2 \pi / k} e^{i n y} d y=\frac{k}{2 \pi i n}\left(e^{2 \pi i n / k}-1\right) .
$$

We can now state the following discrete form of the cardinal series for functions $f \in l^{2}$ such that $f^{\prime}(x)=\sum e^{-i n x} f(n)=0$ for $x \notin(0,2 \pi / k)$ :

$$
f(m)=\frac{k}{2 \pi} \sum f(k n) \frac{e^{2 \pi i(m-n k) / k}-1}{i(m-k n)} .
$$

Mugler (1980) has given a rather different discrete analogue of the cardinal series that is closely connected with a discrete version of the Paley-Wiener theorem.

EXAMPLE 5. Let $G$ be $\Delta$, the dyadic group of all $0-1$ sequences $\left(t_{j}\right)=$ $\ldots ; 0, t_{-N}, \ldots, t_{0} . t_{1}, \ldots$ that are 0 -stationary to the left, under "no carry" binary addition.

Let $t=\left(t_{j}\right)$ and $x=\left(x_{j}\right)$ be two members of $\Delta$. Then the Walsh function $\psi_{t}(x)$ is defined by

$$
\psi_{t}(x)= \pm 1 \text { according as } \sum t_{1-n} x_{n} \text { is even or odd. }
$$

It is well known that the Walsh functions constitute the character group $\Gamma$ of $\Delta$ (see, e.g., Pichler (1973, p. 26) and the references given there). Next let $k$ be a 
fixed positive integer and put $H=\left\{s / 2^{k}, s=0,1, \ldots\right\}$, where $s / 2^{k}$ is given its finite dyadic expansion. $H$ is a discrete subgroup of $\Delta$.

The annihilator $\Lambda$ of $H$ is isomorphic to the set of all 0-1 sequences $\lambda$ such that $\lambda=\ldots, \lambda_{-k}, 0, \ldots, 0.0, \ldots$, for then if $x \in H, \sum \lambda_{1-n} x_{n}=0$ and $\psi_{\lambda}(x)=$ 1. As usual, we take $\Omega=\Gamma / \Lambda$, and it follows that we can let $\Omega=\left[0,2^{k}\right)$. Then

$$
\phi(x)=2^{-k} \int_{0}^{2^{k}} \psi_{r}(x) d r= \begin{cases}1, & x \in\left(0,2^{-k}\right) \\ 0, & \text { otherwise. }\end{cases}
$$

For this calculation see Splettstösser (1980, p. 366).

We now now state the sampling theorem in its Walsh-Fourier analysis setting:

Let $f \in L^{2}(\Delta)$ and

$$
f(t)=\int_{0}^{2^{k}} \psi_{r}(t) f(r) d r
$$

Then

$$
f(t)=\sum_{s=0}^{\infty} f\left(\frac{s}{2^{k}}\right) \chi\left(s 2^{-k},(s+1) 2^{-k}\right) .
$$

The kind of functions appearing in this theorem, namely those whose Walsh-Fourier transforms have support on $\left[0,2^{k}\right)$, are called sequency bandlimited in engineering terminology. They are important for certain applications, as can be gathered from the references given in this section. It might be mentioned that sequency band-limited functions are step functions, as one deduces from the theorem; but this is a reversal of the usual procedure for proving it.

This Walsh sampling theorem seems to be due originally to Pichler (see, e.g., Pichler (1973) and the references to his earlier work cited there). We have also mentioned (\$3.3) that Kak deduced the theorem from Kramer's generalised sampling theorem. Several others have taken a hand in the introduction of the theorem; D. K. Cheng and D. L. Johnson (1973) use the $\delta$ method of derivation and refer to Johnson's thesis as their primary source; see also Maqusi (1972) and Kawamura and Tanaka (1978).

Several other studies of this kind of sampling theorem for sequency-limited functions have appeared recently; see, e.g., the bibliography in Butzer (1983). Ziegler (1981) has given a version in the context of Haar-Fourier analysis.

EXAMPLE 6. In the last example of the present section we shall see how a multidimensional form of the ordinary cardinal series can be obtained from Kluvánek's theorem by letting $G$ be $\mathbb{R}^{N}$. A more detailed survey of multidimensional forms will be found in Story 5 .

Let the vectors $\left\{v_{j}\right\}, j=1,2, \ldots, N$, constitute a basis for $\mathbb{R}^{N}$, and let $\left\{u_{j}\right\}$ be the biorthogonal set to $\left\{v_{j}\right\}$ such that $v_{i} \cdot u_{j}=2 \pi \delta_{i j}$. For $x=$ $\left(x_{1}, x_{2}, \ldots, x_{N}\right) \in \mathbb{R}^{N}$ put

$$
v[x]=v_{1} x_{1}+v_{2} x_{2}+\cdots+v_{N} x_{N}
$$

Then

$$
\mathbb{L}^{N}=\left\{v[m]: m \in \mathbb{Z}^{N}\right\}
$$


is called the sampling lattice. It is clearly a subgroup of $\mathbb{R}^{N}$, and we take this to be $H$ of Kluvanek's theorem.

Now if $m, k \in \mathbb{Z}^{N}$ we have

$$
\exp (i v[m] \cdot u[k])=\exp (2 \pi i m \cdot k)=1 ;
$$

hence,

$$
\Lambda=\left\{\exp (i x \cdot u[k]): k \in \mathbb{Z}^{N}\right\} .
$$

For $\Omega$ we take $\Gamma / \Lambda$, which can be taken as the parallelepiped $U$ with one vertex at the origin whose edges are defined by the vectors $\left\{u_{j}\right\}$. Then

$$
\phi(t)=\int_{U} e^{i t \cdot y} d y
$$

here $d y$ means that the integration is with respect to $N$-dimensional Lebesgue measure.

Kluvánek's theorem now gives us a cardinal series expansion for functions $f \in L^{2}\left(\mathbb{R}^{N}\right)$ whose Fourier transforms are null outside $U$. We get a standard case by choosing $v_{j}=W_{j} e_{j}$, where the $W_{j}$ 's are positive constants and $\left\{e_{j}\right\}$ is the natural basis for $\mathbb{R}^{N}$. This case can be called "rectangular", or "square" if all the $W_{j}$ 's are equal. Then the rectangular cardinal series for $f$ is

$$
f(t)=\sum f\left(\frac{n}{W}\right) \frac{\sin \pi\left(W_{1} t_{1}-n_{1}\right)}{\pi\left(W_{1} t_{1}-n_{1}\right)} \ldots \frac{\sin \pi\left(W_{N} t_{N}-n_{N}\right)}{\pi\left(W_{N} t_{N}-n_{N}\right)} .
$$

\section{STORY FIVE}

\section{EXTENSIONS TO HIGHER DIMENSIONS}

5.1. Foreword. J. M. Whittaker's extension to two dimensions of the ordinary one-dimensional cardinal series involves the Weierstrass $\sigma$-function, as we saw in $\$ 1.4$, and it seems clear that the intention was to find an extension that would remain firmly within the framework of classical function theory. A particular feature of the one-dimensional case is thus preserved, but others, such as the Nyquist sampling rate, the band-limited nature of the sum, etc., are at best unaccounted for and perhaps lost altogether.

Other known extensions to higher dimensions follow a similar pattern. In each case it is possible to discern some property in one dimension that is being given a multidimensional analogue, but at the same time it seems that no higher-dimensional form preserves all the desirable features of the one-dimensional case.

More remarks will be made about this in context.

5.2. The $K$-cardinal series. In $R$. P. Gosselin's multidimensional form of the cardinal series, it is the orthogonality of the translates of $(\sin \pi x) / \pi x$ over the integers that is given a multidimensional analogue. One considers a function $K$ 
of $L^{2}\left(\mathbb{R}^{N}\right)$ for which $\left\{K(x+n): n \in \mathbb{Z}^{N}\right\}$ is orthogonal in $L^{2}\left(\mathbb{R}^{N}\right)$. Then

$$
\sum a_{n} K(x+n), \text { where } a_{n}=\int_{\mathbf{R}^{N}} f(x) K(x+n) d x,
$$

is called the $K$-cardinal series for $f$.

The proofs of the next three theorems follow standard $L^{2}$ methods and are found in Gosselin (1963). The first thing to do is characterise that class of functions $K$ whose translates are indeed orthogonal in the sense described above.

THEOREM. If $g$ belongs to $L\left(\mathbb{R}^{N}\right)$ and

$$
\sum|g(x+2 \pi n)|=\text { constant } \text { a.e., }
$$

then the function $K$ whose Fourier transform is $|g(x)|^{1 / 2} e^{i \phi(x)}, \phi$ real and measurable, is an $L^{2}$ function whose translates are orthogonal.

On the other hand, if the $L^{2}$ function $K$ has orthogonal translates then

$$
\sum\left|K^{\top}(x+2 \pi n)\right|^{2}=\text { constant a.e. }
$$

Some further interesting facts about $K$-cardinal series are contained in the following two theorems.

TheOREM. (a) Let $T_{K}$ denote the closed linear span in $L^{2}\left(\mathbb{R}^{N}\right)$ of the orthogonal set $\left\{K(x+n): n \in \mathbb{R}^{N}\right\}$. Then the $L^{2}$ function $f$ belongs to $T_{K}$ if and only if $f^{\wedge}$ $=K^{\wedge} F^{*}$, where

$$
F^{*}=(2 \pi)^{N} \sum f^{\mathcal{Y}}(x+2 \pi n) \overline{K^{\mathcal{Y}}(x+2 \pi n)} .
$$

(b) If in part (a) supp $K^{\wedge}$ is compact, this condition is equivalent to

$$
f(x)=\int_{\mathbf{R}^{N}} f(t) L(x, t) d t,
$$

where

$$
L(x, t)=\sum e^{2 \pi i n \cdot x} \int_{\mathbf{R}^{N}} K(u) \overline{K(u+t-x)} e^{-2 \pi i n \cdot u} d u
$$

This goes some way towards generalising Hardy's one-dimensional result ( $\mathrm{H} 2$ of $\$ 1.4$, et seq.), but although there are various known multidimensional forms of the Paley-Wiener theorem, there seems to be nothing in the literature to place the present situation in the context of a multidimensional Paley-Wiener space. Gosselin does not use the phrase "reproducing kernel" any more than Hardy did, but I. I. Hirschmann (1964) has pointed out that more could be said about the mode of convergence of the $K$-cardinal series, and presumably the presence of a reproducing kernel was part of what he had in mind. As in the one-dimensional case, it follows that $f$ 's as in part (b) above are represented by their $K$-cardinal series not only in the norm of $L^{2}\left(\mathbb{R}^{n}\right)$, but also pointwise and uniformly on compact subsets of $\mathbb{R}^{n}$.

The following theorem gives a condition for $T_{K}$ to be translation invariant. 
TheOREM. Let $f(x) \in T_{K}$. Then $f(x+\alpha) \in T_{K}\left(\alpha \in \mathbb{R}^{n}\right)$ if and only if $|\hat{K}|=(2 \pi)^{-n / 2}$ on its support.

Compare Weston's result for PW (second theorem (part 2) of §3.2).

Gosselin (1972) went on to make an interesting application of the square cardinal series to the theory of singular integral operators of Calderón-Zygmund type. To begin with, E. C. Titchmarsh (1926a) had shown how the $L^{p}$ boundedness and the inversion and Parseval type formulae for the Hilbert transform could be obtained from similar properties of the discrete analogue:

$$
b_{k}=\frac{\sin \pi \lambda}{\pi} \sum \frac{a_{n}}{k+n+\lambda},
$$

where $\left(a_{n}\right) \in l^{p}$. Then, for example, $\left(b_{k}\right)$ is a bounded operator on $l^{p}$, and

$$
a_{j}=\frac{\sin \pi \lambda}{\pi} \sum \frac{b_{n}}{j+n+\lambda} \text {. }
$$

These operators are clearly forms of the cardinal series, but Titchmarsh did not point this out explicitly; he only used them subsequently in the case $\lambda=1 / 2$. The required properties of the Hilbert transform are obtained by subjecting these discrete formulae to a suitable limiting process.

In the same vein, Gosselin constructed the kernel $K$ of a convolution operator $T$ as a cardinal series

$$
K(x)=\sum^{\prime} K_{0}(n) \prod_{j=1}^{N} \frac{\sin \pi\left(x_{j}+n\right)}{\pi\left(x_{j}+n\right)},
$$

where $K_{0}$ is a Calderón-Zygmund kernel. Then a special sequence of operators constructed from $K$ converges to $K_{0}$ in a weak sense.

5.3. Cardinal series over a general lattice. We continue to discuss the multidimensional cardinal series which constituted the last example of Story 4. Probably the most important feature of this general lattice sampling is that which allows some flexibility in improving the sampling rate over that which is available in the rectangular case. One achieves this by arranging copies of the "band region" $\mathscr{B}$, that subset of $\mathbb{R}^{N}$ over which $f^{\wedge}$ is nonvanishing, in a configuration of closest packing; this in turn minimises the density of the sampling points, and we get a kind of analogue of the Nyquist sampling rate. These matters will be illustrated with a special case, called "hexagonal", which has been closely studied in the literature.

First, we note that, whether $\Omega$ is chosen to be $\Gamma / \Lambda$ or not, Kluvánek's theorem (\$4.1) requires that it be a regular tessellating figure for $\Gamma \equiv \mathbb{R}^{N}$ (regular in the sense that copies of it tessellate $\mathbb{R}^{N}$ by translation over the vectors $\left\{u_{j}\right\}$ ).

Second, in applications $\mathscr{B}$ will probably be fixed in advance and unlikely to furnish the kind of $\Omega$ just described. The best we can do is to enclose $\mathscr{B}$ in a suitable $\Omega$. The choice of $\Omega$ will affect the geometry of the sampling lattice $\mathbb{L}^{N}$, and a sensible choice will be one which minimises the density of $\mathbb{L}^{N}$. Since we 
can associate each point of $\mathbb{L}^{N}$ with a distinct translate of $V(V$ is that parallellepiped with edges determined by the vectors $\left\{v_{j}\right\}$ (see Figure 1)), this is equivalent to maximising the hypervolume $|V|$. This in turn is equivalent to minimising the hypervolume $|U|$ (similarly associated with $\left\{u_{j}\right\}$ ), since one easily shows that $|V|$ is inversely proportional to $|U|$. The ease with which $\Omega$ can be chosen will depend very much on the shape of $\mathscr{B}$.

EXAMPLE. Hexagonal sampling. Take $n=2$ and $\mathscr{B}$ to be the unit disc of $\mathbb{R}^{2}$.

We choose $u_{1}$ and $u_{2}$ so that translated copies of the circular band region are close-packed, and we let $\Omega$ be the hexagonal Dirichlet region associated with this close packing. The density of $\mathbb{L}^{2}$ is $1 /|V|=\sqrt{3} / 2 \pi^{2}$.

Clearly, it would have been less efficient, as far as the density of $\mathbb{L}^{2}$ is concerned, if we had enclosed $\mathscr{B}$ in a square $\Omega$. Then closest packing would not have been achieved, the square two-dimensional cardinal series would have resulted, and the corresponding $\mathbb{L}^{2}$ would have had density $1 / \pi^{2}$. On comparing these two densities one finds that the hexagonal method represents a saving of some 13.4 percent of the points necessary in the square case. On the other hand, the reconstruction function for the hexagonal scheme, which must be calculated by integrating $e^{i t \cdot x}$ over the hexagon of Figure 2, is

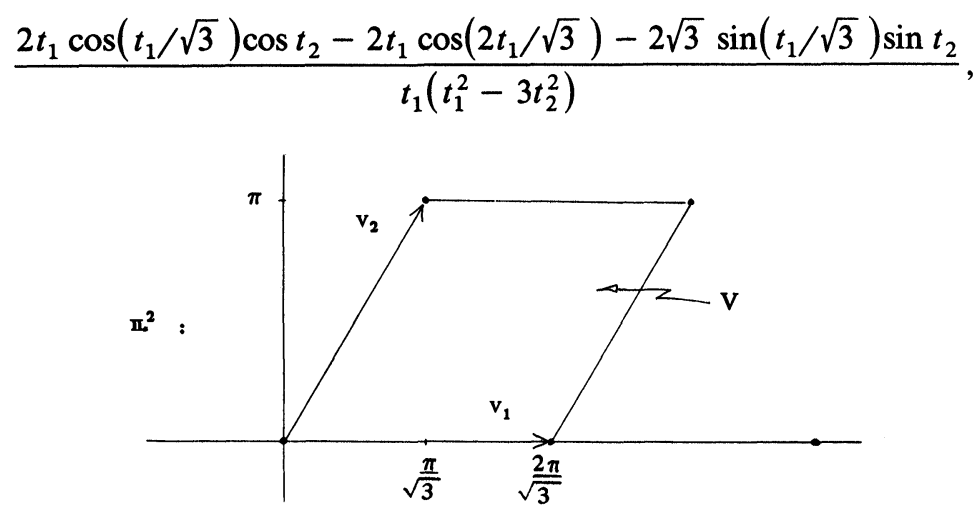

Figure 1

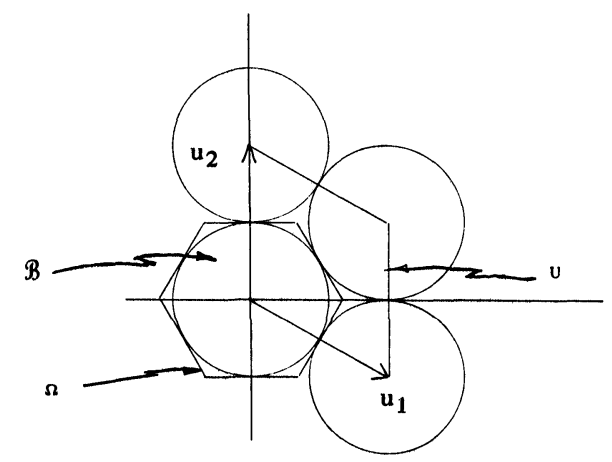

FIGURE 2 
whereas that for the square form is just

$$
\frac{\sin \pi t_{1}}{\pi t_{1}} \cdot \frac{\sin \pi t_{2}}{\pi t_{2}}
$$

Of course, an engineer would have to carefully assess the advantages and disadvantages before adopting one or other of these sampling schemes to solve a practical problem. By and large it seems that electrical engineers prefer the simplicity of the square form or the marginally less simple rectangular form. However, the hexagonal and other nonrectangular forms have been championed by Mersereau (1979) and Mersereau and Speake (1983); they point out that another advantage of the hexagonal scheme, which can be important in certain applications to image processing (Mersereau $(1979$, p. 932)), is that the sample points each have six nearest neighbours; this is convenient for implementation and not enjoyed by the rectangular arraangement. In giving details of hexagonal sampling and several higher-dimensional analogues of it, Petersen and Middleton (1962, p. 286) attribute its introduction to Myakawa.

It is worth mentioning that the reconstruction function given by Kluvanek's theorem is just that which Petersen and Middleton singled out from among other possibilities and called canonical. These other possibilities arise because of the " $\delta$-method" of derivation used by Petersen and Middleton (op. cit., p. 288) that involves the multidimensional analogue of "spectrum repetition", which was such a striking feature of the one-dimensional formulation ( $\$ 2.2)$ :

$$
f^{\mathcal{Y}}(t)=\frac{\phi^{\mathcal{Y}}(t)}{|V|} \sum f^{\mathcal{Y}}(t+u[n])
$$

This certainly requires $\phi^{\wedge}$ to be constant over the band region $\mathscr{B}$, but in that part of $\mathbb{R}^{N}$ not covered by $\mathscr{B}$ and its translates (see Figure 2 , for example), the value of $\phi^{\wedge}$ is arbitrary. We can, if we like, form another kind of reconstruction function by taking

$$
\phi(t)=\text { (const) } \int_{\mathscr{B}} e^{i x \cdot t} d x .
$$

In the cases of a radially symmetric $f$ band-limited to the unit hypersphere centered at the origin of $\mathbb{R}^{N}$, the reconstruction series reduces to a relatively simple form. Indeed, the integral (29) reduces by a very well-known construction to a Bessel function, and the series for such $f$ is

$$
f(x)=|V| \sum_{l \in \mathbf{L}^{N}} f(l) \frac{J_{N / 2}(|x-l|)}{(2 \pi|x-l|)^{N / 2}},
$$

where the $\mathbb{L}^{N}$ is that lattice generated by $\left\{v_{j}\right\}$ for which the corresponding $\left\{u_{j}\right\}$ defines the lattice of centres of hyperspheres in the closest lattice packing configuration. The appropriate lattices for dimensions two through eight are quoted from results of Coxeter by Petersen and Middleton (1962, p. 314).

5.4. Remarks. 1. It seems to have been E. Parzen (1956) who first suggested the rectangular multidimensional form of the cardinal series. Although a special case of more general forms, it has received sporadic attention in its own 
right since that time. For example, Prosser (1966) treated the truncation error in both the $L^{2}$ and $L^{\infty}$ norms, and Splettstösser (1982) has treated the aliasing error and given other reconstruction series (not of cardinal type) which have better aliasing errors.

2. A somewhat different approach to two-dimensional sampling, involving polar coordinates, has been developed for use in optical science. The general idea is this. Suppose that

$$
f(r, \theta)=\sum e^{i n \theta} c_{n}(r),
$$

where

$$
c_{n}(r)=\frac{1}{2 \pi} \int_{0}^{2 \pi} f(r, \theta) e^{-i n \theta} d \theta .
$$

Let us formally calculate the Fourier transform of $f$ term by term; we get

$$
f^{\mathcal{Y}}(\rho, \phi)=\sum e^{-i n \phi} \int_{0}^{\infty} r c_{n}(r) \int_{0}^{2 \pi} \exp (n \theta-r \rho \sin \phi) d \theta d r .
$$

It comes as no surprise that a Bessel function appears here, the second integral being one of the standard representations for $J_{n}(r \rho)$. Thus, we have

$$
f^{\mathcal{Y}}(\rho, \phi)=2 \pi \sum e^{-\ln \phi} \rho^{-1 / 2} \mathscr{H}_{n}\left[r^{1 / 2} c_{n}(r)\right](\rho),
$$

where $\mathscr{H}_{n}$ denotes the Hankel transform (23).

Next, suppose that $f$ is band-limited to the unit disc of $\mathbb{R}^{2}$; then each of the Hankel transforms in (32) vanishes outside [0,1], and, therefore, because of the self-inverse nature of $\mathscr{H}_{n}, r^{1 / 2} c_{n}(r)$ has a representation by the Bessel-Hankel form of the cardinal series (24) with $\nu=n$. This in turn means that $c_{n}(r)$ is determined by the set of samples $\left\{c_{n}\left(j_{n i}\right)\right\}$ - that is, from (31),

$$
c_{n}\left(j_{n i}\right)=\frac{1}{2 \pi} \int_{0}^{2 \pi} f\left(j_{n i}, \theta\right) e^{-i n \theta} d \theta,
$$

in which $j_{n i}$ is the $i$ th zero of the $n$th Bessel function $J_{n}(x)$. Then (30) gives a double series reconstruction for $f$ from information taken from function values on a doubly infinite set of circles which are one-dimensional subsets of $\mathbb{R}^{2}$, rather than from values on the doubly infinite set of points used in the two-dimensional Cartesian approach to the cardinal series.

This form of reconstruction series has been studied and applied by Blažek (1974, 1976). Another polar form has been given by Stark (1979) and Stark and Sarna (1979), which is valid for functions $f$ that are finite Fourier series, and proceeds via finite trigonometric interpolation.

How far can one go in this direction? How can one reconstruct functions defined on $\mathbb{R}^{N}$ and satisfying some sort of band-limited criteria from information such as the function's values on a collection of $M$-dimensional subsets of $\mathbb{R}^{N}$ ? It is an open question.

3. It should be mentioned that multidimensional extensions have been made in connection with the Kramer series, derivative sampling, and jittered sampling; see Jerri (1977) for references. See also Butzer and Engels (1982) for a multidimensional Walsh-type sampling series. 
4. Finally, it is fair to say that multidimensional sampling theory is still in a tentative state of development. For the mathematician the main interest is in theorems giving the existence of cardinal type series representations with good convergence criteria and their mathematical properties; but there is a lack of such theorems in the present literature. More often than not, the mode of convergence for the multisums is not even specified; exceptions are Gosselin (1963) ("spherical" partial sums) and Splettstösser (1982) ("square" partial sums). To my knowledge no extension of the cardinal series to $\mathbb{C}^{N}$ has ever been made.

Here is an area where much remains to be done.

\section{CONCLUSION}

In concluding this survey of matters relating to the cardinal series, I would like to include four more topics which are of sufficient interest to warrant a brief description.

I. The first of these topics does not involve the cardinal series directly, but is of interest in the study of band-limited functions in that it concerns reconstruction from samples taken at points intrinsic to the function itself, in this case its real zeros. This arose as an engineering problem in the context of speech transmission when it became recognised that it was efficient to transmit the "clipped" version sgn $f(t)$ of a speech signal $f$, and then reconstitute $f$ as best one can at the receiving point. For an account of the origins of this problem and the first mathematical treatment of it, see Bond and Cahn (1958).

An example, or rather a family of examples, will help to illustrate the problem.

EXAMPLE. If $m$ is an even integer exceeding two, then

$$
-\int_{-\infty}^{t} \frac{\sin ^{m}(\pi u / m)}{(\pi \cdot u / m)^{m-1}} d u
$$

belongs to $\mathrm{PW}$ and is positive everywhere on $\mathbb{R}$.

For $m=4$, the example was given by Khurgin and Yakovlev (1977). The positivity is obvious; as to membership of $\mathrm{PW}$, the Fourier transform is simply related to, and indeed has the same support as, that of $\left[(\pi u / m)^{-1} \sin (\pi u / m)\right]^{m}$, which is available in standard lists of transforms (e.g., Oberhettinger (1957, p. 19)).

It is clear from this example that there is no hope, in general, for reconstructing a band-limited signal from knowledge of its real zeros alone. But if we consider the natural extension of $f$ to the complex plane, the situation is just the opposite; among the many classical theorems on the zeros of what we now call band-limited functions (see, e.g., Boas (1954, Chapter 8)), one of Titchmarsh's (1926) is appropriate here. It guarantees that a band-limited function has infinitely many zeros, and these serve to determine it via a product representation. This is not very convenient for engineers, however, who do not expect to be able to detect complex zeros in their signals. Much ingenuity has gone into circumventing this difficulty. See Jerri (1977, §IVF) for references to 
the older literature, and E. Masry (1982) and the references given there for a more recent approach involving the comparison of a band-limited signal with a random reference, or "dither", signal.

II. Zakai (1965) has generalised the notion of band-limited function by calling $f$ "band-limited $(V, 2 \varepsilon)$ " if it is reproduced by convolution, not with the ordinary kernel $(\sin \pi t) / \pi t$ as for Paley-Wiener functions, but with the more general kernel

$$
\left(\frac{2}{\pi}\right)^{1 / 2} \frac{\sin (V+\varepsilon) t}{t} \frac{\sin \varepsilon t}{\varepsilon t} .
$$

This function is the inverse Fourier transform of that continuous function which vanishes outside $[-V-2 \varepsilon, V+2 \varepsilon]$, is constantly equal to one on $[-V, V]$, and is linear on $[-(V+2 \varepsilon),-V]$ and $[V, V+2 \varepsilon]$.

Zakai shows that a function $f$ that is band-limited in this sense, and is such that $f(t) /\left(1+t^{2}\right)^{1 / 2} \in L^{2}(\mathbb{R})$, is necessarily of the form $f(0)+\operatorname{tg}(t), g \in$ $\mathrm{PW}_{(V+2 \varepsilon)}$. Such functions are represented by ordinary scaled cardinal series, the positive scale factor being less than $\pi / V$, with uniform convergence on compact subsets of the complex $t$-plane. One shows this by giving $f$ its representation in (scaled) $T$-cardinal series (see \$1.4). A few computational devices then serve to show that the $T$-cardinal series reduces to an ordinary cardinal series.

III. Ryavec (1979) has given a form of the cardinal series in which the sampling points are the nonreal zeros of the Riemann zeta function $\zeta(z)$. It is based on a summation formula of $A$. Weil, which involves summing the Fourier transform of a function over, essentially, the zeros of $\zeta$, a situation which is, of course, similar in spirit to that which relates the ordinary cardinal series to the Poisson summation formula. Ryavec deduced a new representation for $\zeta$ from this kind of cardinal series, which involves a summation over its zeros.

IV. Throughout the preceding pages we have become used to the idea that the Nyquist sampling rate is the minimum rate at which a band-limited function can be sampled and reconstructed by the cardinal series, and indeed by other related series. But the question can be raised as to whether this rate might be capable of improvement in some way. An important study of this problem was made by Landau (1967a). He says (p. 1701) “... it is conceivable that signals might be recoverable from their values taken at a lower rate, if the sampling instants were chosen differently; or if the signals had their frequencies in a union of several bands; or at the cost of more computing...", but goes on to show that, in effect, the Nyquist rate cannot be bettered.

Let $\mathrm{PW}_{s}$ denote that subset of $L^{2}(\mathbb{R})$ whose members have Fourier transforms supported on $S$, and let $\left(t_{n}\right), n \in \mathbb{Z}$, be a sequence of points of $\mathbb{R}$ which are separated by a least positive distance $d$. Then $\left(t_{n}\right)$ is called a set of uniqueness for $\mathrm{PW}_{S}$ if $f\left(t_{n}\right)=0$ implies $f \equiv 0$ for every $f \in \mathrm{PW}_{S}$. Again, $\left(t_{n}\right)$ is called a set of stable sampling for $\mathrm{PW}_{S}$ if there exists a positive constant $K$ such that, for every $f \in \mathrm{PW}_{S},\|f\|^{2} \leqslant K \Sigma\left|f\left(t_{n}\right)\right|^{2}$. This very familiar kind of condition guarantees that small errors in the samples will cause correspondingly small errors in the reconstructed function. Clearly, such a set is essential for applications; it will be a set of uniqueness, but not conversely. 
Beurling and Malliavin have shown that a least upper bound can be constructed for those bandwidths for which a given $\left(t_{n}\right)$ is a set of uniqueness. Landau showed that stable sampling cannot be performed at a rate lower than Nyquist, and dually, that data cannot be transmitted as samples at a rate higher than Nyquist. See also Landau (1967b).

Many questions for further investigation will, no doubt, have occurred to the interested reader. We have noted various classes of functions which can be represented by their cardinal series; an interesting question would be to ask for a classification of all such functions. Similar questions would arise for allied series and for multidimensional representations.

\section{ADDENDA}

It is a pleasure to thank Professors J. L. Brown, P. L. Butzer, E. Masry and W. Schempp for bringing me up to date on certain topics arising in the stories just concluded. Their comments reached me only after the manuscript had received final preparation.

Professor Brown informs me that he too has been troubled by the "Cauchy connection" mystery (see \$1.2), especially in view of a recent article in the Sunday New York Times of 1 January 1984 by Hans Fantel: “Will disks still spin in 2020?" Here one can read that the concept of substituting a numerical code for "wiggly waveforms... actually dates back to Napoleonic times when the Baron Augustin Cauchy devised a theory for describing wave phenomena through mathematical sampling". Apparently no source for this assertion has so far come to light; nevertheless, in view of the fact that Cauchy was one of the instigators of trigonometrical interpolation (along with several others writing at about that time; see Burkhardt (1899-1916)), it is not without some justification.

To be more specific, Brown has pointed out that Cauchy had developed some quite general finite sampling formulae in the second part of his 1841 paper (op. cit,) which are applicable to trigonometrical polynomials, or "harmonic-limited" functions in Brown's phrase, and which incidentally were rediscovered not long ago. See his preprint Cauchy and polar sampling theorems, in which he makes several remarks about the "mystery" and goes on to give a more general finite sampling formula.

On the other hand, I have concentrated on the first part of the 1841 paper in $\S \S 1.2$ and 4.2, where Cauchy's finite series associated with torus groups are described, since it is here that we find his contributions to the theory of cardinal series. Of course, the problem concerning H. S. Black's citation remains unresolved.

Professor Butzer informs me that he and his colleagues at Aachen have recently proved that the cardinal series, the Poisson summation formula, the Euler-MacLaurin summation formula, and Cauchy's integral formula are basically equivalent. See P. L. Butzer, S. Ries, and R. L. Stens, Shannon's sampling theorem, Cauchy's integral formula and related results, Arbeitsbericht, 1984 , and the references given there.

Of particular interest among the helpful comments sent by Professor Masry is the fact that Zakai's class of band-limited functions (described in part II of 
the present Conclusion) does not depend on the parameter $\varepsilon$, as might appear at first sight; indeed, he and S. Cambanis have verified Zakai's conjecture that $f$ belongs to this class if and only if $f(t)=f(0)+t g(t)$, where $g$ belongs to $\mathrm{PW}_{V}$. Such a function is evidently band-limited in the sense of distributions (see §3.5). See the references given in A. J. Lee, Characterisation of bandlimited functions and processes, Inform. and Control 31 (1976), 258-271, where the Zakai class and its characterisation (above) are generalised; Lee shows that the members of this more general class are represented by scaled cardinal series modified by the insertion of a convergence enhancing factor. This provides more information regarding the first question raised at the end of $\S 3.5$ on distributions.

Professor Schempp has kindly pointed out to me that the general Poisson summation formula (\$2.5) has deep geometric roots in harmonic analysis of the compact Heisenberg nilmanifold; for background see, for example, the survey by $\mathrm{R}$. Howe, On the role of the Heisenberg group in harmonic analysis, Bull. Amer. Math. Soc. (N.S.) 3 (1980), 821-843.

The Heisenberg nilpotent group provides the natural mathematical structure for describing the connection between quantum mechanics and information theory; actually, this had been anticipated by J. D. Weston (see the quotation in \$1.5). In particular, the Fourier cotransform has, in this setting, a factorisation called the Poisson-Weil factorisation, in terms of the Weil-Brezin automorphism. Professor Schempp has obtained the classical cardinal series as a special case of this. See W. Schempp, Gruppentheoretische Aspekte der Signalubertragung und der kardinal Interpolatiossplines. I, Math. Methods Appl. Sci. 5 (1983), 195-215.

Important applications in signal processing stem from this approach, particularly to the design of radar systems. See W. Schempp, Radar ambiguity functions, nilpotent harmonic analysis, and holomorphic theta series, Special Functions: Group Theoretical Aspects and Applications (R. A. Askey et al., eds.), Reidel, 1984. See also the many references given there.

I also thank Professor Schempp for pointing out to me another application of cardinal series interpolation-to medical tomography. See G. Schwierz, W. Härer and K. Wiesent, Sampling and discretization problems in X-ray-CT, Mathematical Aspects of Computerized Tomography, Springer-Verlag, Berlin and New York, 1981.

\section{REFERENCES}

A. V. Balakrishnan (1957), A note on the sampling principle for continuous signals, IRE Trans. Inform. Theory IT-3, 143-146.

A. B. Bhatia and K. S. Krishnan (1948), Light-scattering in homogeneous media regarded as reflexion from appropriate thermal elastic wave, Proc. Roy. Soc. London Ser. A 192, 181-194.

C. Bigelow and D. Day (1983), Digital typography, Scientific American (2) 249, 94-105.

H. S. Black (1953), Modulation theory, van Nostrand, Princeton, N. J.

V. Blažek (1974), Sampling theorem and the number of degrees of freedom of an image, Optics Comm. 11, 144-147. (1976), Optical information processing by the Fabry-Perot resonator, Optical Quantum Electronics 8, 237-240.

R. P. Boas, Jr. (1954), Entire functions, Academic Press, New York. 
(1972), Summation formulas and band-limited signals, Tôhoku Math. J. 24, 121-125.

R. P. Boas, Jr. and H. Pollard (1973), Continuous analogs of series, Amer. Math. Monthly 80, 18-25.

F. E. Bond and C. R. Cahn (1958), On sampling the zeros of bandwidth limited signals, IRE Trans. Inform. Theory IT-4, 110-113.

E. Borel (1897), Sur l'interpolation, C. R. Acad. Sci. Paris 124, 673-676. (1898), Sur la recherche des singularités $d$ 'une fonction définie par un développement de

Taylor, C. R. Acad. Sci. Paris 127, 1001-1003. (1899), Mémoire sur les séries divergentes, Ann. École Norm. Sup. (3) 16, 9-131.

J. L. Brown, Jr. (1967), On the error in reconstructing a non-bandlimited function by means of the bandpass sampling theorem, J. Math. Anal. Appl. 18, 75-84; Erratum, ibid. 21 (1968), 699.

(1980), First order sampling of bandpass signals - a new approach, IEEE Trans. Inform.

Theory IT-26, 613-615.

CAS-28, 101-106.

(1981), Multi-channel sampling of low-pass signals, IEEE Trans. Circuits and Systems

T. A. Brown (1915-1916), Fourier's integral, Proc. Edinburgh Math. Soc. 34, 3-10.

H. Burkhardt (1899-1916), Trigonometrische Interpolation, Enzyklopädie Math. Wiss. IIA9a, Teubner, Leipzig.

P. L. Butzer (1983), A survey of the Whittaker-Shannon sampling theorem and some of its extensions, J. Math. Res. Exposition 3, 185-212.

P. L. Butzer and W. Engels (1982), Dyadic calculus and sampling theorems for functions with multi-dimensional domain. I, II, Inform. and Control 52, 333-363.

P. L. Butzer and R. Nessel (1971), Fourier analysis and approximation, Vol. 1, Academic Press, New York and London.

P. L. Butzer and W. Splettstösser (1977), Approximation und interpolation durch verallgemeinerte Abtastsummen, Westdeutscher Verlag, Opladen.

P. L. Butzer and R. Stens (1983), The Euler-MacLaurin summation formula, the sampling theorem, and approximate integration over the real axis, Linear Algebra Appl. 52 / 53, 141-155.

L. L. Campbell (1968), Sampling theorem for the Fourier transform of a distribution with bounded support, SIAM J. Appl. Math. 16, 626-636.

M. L. Cartwright (1936), On certain integral functions of order one, Quart. J. Math. Oxford Ser. 7, 46-55.

A. L. Cauchy (1841), Mémoire sur diverses formules d'analyse, C. R. Acad. Sci. Paris 12, 283-298.

P. Cazzaniga (1882), Esspressione di funzioni intere che in posti dati arbitrariamente prendono valori prestabiliti, Ann. Mat. Pura Appl. (2) 10, 278-290.

D. K. Cheng and D. L. Johnson (1973), Walsh transform of sampled time functions and the sampling principle, Proc. IEEE 61, 674-675.

R. R. Coifman and G. Weiss (1977), Extensions of Hardy spaces and their use in analysis, Bull. Amer. Math. Soc. 83, 569-645.

W. L. Ferrar (1925), On the cardinal function of interpolation-theory, Proc. Roy. Soc. Edinburgh 45, 267-282.

323-333.

(1926), On the cardinal function of interpolation-theory, Proc. Roy. Soc. Edinburgh 46,

L. J. Fogel (1955), A note on the sampling theorem, IRE Trans. Inform. Theory 1, 47-48.

C. F. Gauss (1900), Carl Friedrich Gauss Werke, Band 8, Königl. Gesellschaft Wiss. Gottingen, Teubner, Leipzig.

S. Goldman (1953), Information theory, Constable, London.

E. A. Gonzáles-Velasco and E. Sanvicente (1980), The analytic representation of bandpass signals,

J. Franklin Inst. 310, 135-142.

R. P. Gosselin (1963), On the $L^{p}$ theory of cardinal series, Ann. of Math. (2) 78, 567-581. (1972), Singular integrals and cardinal series, Studia Math. 44, 39-45.

M. Guichard (1884), Sur les fonctions entières, Ann. Sci. École Norm. Sup. (3) 1, 427-432.

J. Hadamard (1901), La serie de Taylor et son prolongement analytique, Scientia 12, 1-100.

A. H. Haddad, K. Yao and J. B. Thomas (1967), General methods for the derivation of sampling theorems, IEEE Trans. Inform. Theory IT-13, 227-230. 
G. H. Hardy (1941), Notes on special systems of orthogonal functions. IV: The orthogonal functions of Whittaker's cardinal series, Proc. Cambridge Philos. Soc. 37, 331-348.

J. R. Higgins (1972), An interpolation series associated with the Bessel-Hankel transform, J. London Math. Soc. 5, 707-714. (1976), A sampling theorem for irregularly spaced sample points, IEEE Trans. Inform. Theory IT-22, 621-622. (1977), Completeness and basis properties of sets of special functions, Cambridge Univ. Press, Cambridge.

I. I. Hirschman (1964), Review of "On the $L^{p}$ theory of the cardinal series" by $R$. $P$. Gosselin, Math. Rev. 27 \# 6086, 1163.

D. L. Jagerman and L. J. Fogel (1956), Some general aspects of the sampling theorem, IEEE Trans. Inform. Theory 2, 139-156.

A. J. Jerri (1977), The Shannon sampling theorem-its various extensions and applications: $a$ tutorial review, Proc. IEEE 65, 1565-1596.

P. E. B. Jourdain (1905), On the general theory of functions, J. Reine Angew. Math. 128, 169-210.

S. C. Kak (1970), Sampling theorem in Walsh-Fourier analysis, Electronics Lett. 6, 447-448.

M. Kawamura and S. Tanaka (1978), Proof of sampling theorem in sequency analysis, Systems-Comput.-Controls 9, 10-15.

Y. I. Khurgin and V. P. Yakovlev (1977), Progress in the Soviet Union on the theory and applications of bandlimited functions, Proc. IEEE 65, 1005-1029.

I. Kluvánek (1965), Sampling theorem in abstract harmonic analysis, Mat.-Fyz. Casopis Sloven. Akad. Vied 15, 43-48. (1972), Positive definite signals with maximal energy, J. Math. Anal. Appl. 39, 580-585.

A. Kohlenberg (1953), Exact interpolation of bandlimited functions, J. Appl. Phys. 24, 1432-1436.

A. N. Kolmogorov (1956), On the Shannon theory of information transmission in the case of continuous signals, IRE Trans. Inform. Theory IT-2, 102-108.

A. N. Kolmogorov and V. M. Tihomirov (1960), $\varepsilon$-Entropie und $\varepsilon$-Kapazität von Mengen in Funktional Räumen, Math. Forschungsberichte 10, VEB Deutscher Verlag Wiss., Berlin. (Translated from the Russian)

V. A. Kotel'nikov (1933), On the carrying capacity of the "ether" and wire in telecommunications, Material for the First All-Union Conference on Questions of Communication, Izd. Red. Upr. Svyazi RKKA, Moscow. (Russian)

H. P. Kramer (1957), A generalised sampling theorem, J. Math. Phys. 63, 68-72.

$275-287$.

(1973), The digital form of operators on band-limited functions, J. Math. Anal. Appl. 44,

H. J. Landau (1967a), Sampling, data transmission, and the Nyquist rate, Proc. IEEE 55, 1701-1706.

(1967b), Necessary density conditions for sampling and interpolation of certain entire functions, Acta Math. 117, 37-52.

H. J. Landau and H. O. Pollack (1962), Prolate spheroidal wave functions, Fourier analysis and uncertainty. III, Bell System Tech. J. 41, 1295-1336.

D. A. Linden (1959), A discussion of sampling theorems, Proc. IRE 47, 1219-1226.

D. A. Linden and N. M. Abramson (1960), A generalisation of the sampling theorem, Inform. and Control 3, 26-31; Errata, Ibid. 4 (1961), 95-96.

H. D. Lüke (1978), Zur Entstehung des Abtasttheorems, Nachr. Techn. Z. 31, 271-274.

A. J. Macintyre (1938), Laplace's transformation and integral functions, Proc. London Math. Soc. 45, 1-20.

W. Magnus, F. Oberhettinger and R. P. Soni (1966), Formulas and theorems for the special functions of mathematical physics, 3rd ed., Springer-Verlag, Berlin.

M. Maqusi (1972), Walsh functions and the sampling principle, Proc. Walsh Functions Sympos., U. S. Naval Research Lab.

E. Masry (1982), The application of random reference sequences to the reconstruction of clipped differentiable signals, IEEE Trans. Acoust. Speech Signal Process. ASSP-30, 953-963.

F. C. Mehta (1975), Sampling expansion for band-limited signals through some special functions, J. Cybernetics 61-68. 
R. M. Mersereau (1979), The processing of hexagonally sampled two dimensional signals, Proc. IEEE 67, 930-949.

R. M. Mersereau and T. C. Speake (1983), The processing of periodically sampled multidimensional signals, IEEE Trans. Acoust. Speech Signal Process. ASSP-31, 188-194.

H. Meschowski (1962), Hilbertsche Räume mit kernfunktion, Springer-Verlag, Berlin.

D. H. Mugler (1976), Convolution, differential equations and entire functions of exponential type, Trans. Amer. Math. Soc. 216, 145-187. (1980), The discrete Paley-Wiener theorem, J. Math. Anal. Appl. 75, 172-179.

J. Neveu (1965), Le problème d'échantillonnage et de l'interpolation d'un signal, C. R. Acad. Sci. Paris 260, 49-51.

K. Ogura (1920), On some central difference formulas of interpolation, Tôhoku Math. J. 17, 232-241.

F. Oberhettinger (1957), Tabellen zur Fourier Transformation, Springer-Verlag, Berlin.

A. Papoulis (1968), Systems and transforms with applications in optics, McGraw-Hill, New York.

E. Parzen (1956), A simple proof and some extensions of sampling theorems, Tech. Rep. 7, Stanford Univ., Stanford.

D. P. Petersen (1963), Sampling of space-time stochastic processes with application to information and design systems, Thesis, Rensselaer Polytechnic Inst., Troy, N. Y.

D. P. Petersen and D. Middleton (1962), Sampling and reconstruction of wave number-limited functions in $\mathrm{N}$-dimensional Euclidean spaces, Inform. and Control 5, 279-323.

F. Pichler (1973), Walsh functions - introduction to the theory, Signal Processing (Proc. NATO Advanced Study Inst. Signal Processing, J. W. R. Griffiths et al., eds.), Academic Press, London and New York, pp. 23-41.

S.-D. Poisson (1820), Mémoire sur la manière d'exprimer les fonctions, par des séries de quantités périodiques, et sur l'usage de cette transformation dans la résolution de différens problèmes, J. École Roy. Polytechnique 11, 417-489.

G. Pólya (1931), Aufgabe 105, Jahresber. Deutsch. Math.-Verein. 40, 80.

R. T. Prosser (1966), A multidimensional sampling theorem, J. Math. Anal. Appl. 16, 574-584.

C. Ryavec (1979), A non-linear analogue of the cardinal series, Quart. J. Math. Oxford Ser. 30, 223-227.

C. E. Shannon (1948), A mathematical theory of communication, Bell System Tech. J. 27, 379-423.

(1949), Communication in the presence of noise, Proc. IRE 37, 10-21.

I. Someya (1949), Waveform transmission, Shukyo, Tokyo.

B. Spain (1940), Interpolated derivatives, Proc. Roy. Soc. Edinburgh 60, 134-140. (1958), Interpolated derivatives, Proc. Edinburgh Math. Soc. 9, 166-167.

W. Splettstösser (1980), Error analysis in the Walsh sampling theorem, IEEE Sympos. Electromagnetic Computibility, Baltimore. Inst. for Electrical and Electronics Engineers, Service Center, Piscataway, N. J., pp. 366-370.

(1982), Sampling approximation of continuous functions with multidimensional domain,

IEEE Trans. Inform. Theory IT-28, 809-814.

W. Splettstösser, R. L. Stens and G. Wilmes (1981), On approximation by the interpolating series of G. Valiron, Funct. Approx. Comment. Math. 11, 39-56.

H. Stark (1979), Sampling theorems in polar coordinates, J. Opt. Soc. Amer. 69, 1519-1525.

H. Stark and C. S. Sarna (1979), Image reconstruction using polar sampling theorems, Appl. Optics 18, 2086-2088.

J. F. Steffensen (1914), Uber eine Klasse von ganzen Funktionen und ihre Anwendung auf die Zahlentheorie, Acta Math. 37, 75-112.

R. L. Stens (1980), Error estimates for sampling sums based on convolution integrals, Inform. and Control 45, 37-47.

M. Theis (1919), Uber eine Interpolations-formeln von de la Vallée Poussin, Math. Z. 3, 93-113.

E. C. Titchmarsh (1926a), Reciprocal formulae involving series and integrals, Math. Z. 25, 321-347.

(1926b), The zeros of certain integral functions, Proc. London Math. Soc. 25, 283-302.

(1948), Introduction to the theory of Fourier integrals, 2nd ed., Clarendon Press, Oxford.

L. Tschakaloff (1933), Zweite Losung der Aufgabe 105, Jahresber. Deutsch. Math.-Verein. 43, 11-12.

G. Valiron (1925), Sur la formule d'interpolation de Lagrange, Bull. Sci. Math. (2) 49, 181-192; 203-224. 
Ch.-J. de la Vallée Poussin (1908), Sur la convergence des formules d'interpolation entre ordonnées equidistantes, Acad. Roy. Belg. Bull. Cl. Sci. 1, 319-410.

J. D. Weston (1949a), The cardinal series in Hilbert space, Proc. Cambridge Philos. Soc. 45, 335-341.

(1949b), A note on the theory of cummunication, Philos. Mag. (303) 40, 449-453.

E. T. Whittaker (1915), On the functions which are represented by the expansions of the interpolation theory, Proc. Roy. Soc. Edinburgh 35, 181-194.

J. M. Whittaker (1929a), On the cardinal function of interpolation theory, Proc. Edinburgh Math. Soc. 1, 41-46. 169-176.

(1929b), On the "Fourier theory" of the cardinal function, Proc. Edinburgh Math. Soc. 1, (1935), Interpolatory function theory, Cambridge Univ. Press, Cambridge.

J. L. Yen (1956), On nonuniform sampling of bandwidth-limited signals, IRE Trans. Circuit Theory CT-3, 251-257.

R. M. Young (1980), An introduction to nonharmonic Fourier series, Academic Press, New York.

M. Zakai (1965), Band-limited functions and the sampling theorem, Inform. and Control 8 , 143-158.

W. Ziegler (1981), Haar-Fourier Transformation auf dem $\mathbf{R}^{+}$, Doctoral Diss. Technische Univ. München.

A. Zygmund (1959), Trigonometric series, Vol. II. 2nd ed., Cambridge Univ. Press, Cambridge.

Department of Science, Cambridgeshire College of Arts and Technology, Cambridge, ENGLAND 
